\title{
A variation of Broyden Class methods using Householder adaptive transforms
}

\author{
Stefano Cipolla*, C. Di Fiore ${ }^{\dagger}$, P. Zellini ${ }^{\dagger}$
}

January 22, 2020

\begin{abstract}
In this work we introduce and study novel Quasi Newton minimization methods based on a Hessian approximation Broyden Class-type updating scheme, where a suitable matrix $\widetilde{B}_{k}$ is updated instead of the current Hessian approximation $B_{k}$. We identify conditions which imply the convergence of the algorithm and, if exact line search is chosen, its quadratic termination. By a remarkable connection between the projection operation and Krylov spaces, such conditions can be ensured using low complexity matrices $\widetilde{B}_{k}$ obtained projecting $B_{k}$ onto algebras of matrices diagonalized by products of two or three Householder matrices adaptively chosen step by step. Extended experimental tests show that the introduction of the adaptive criterion, which theoretically guarantees the convergence, considerably improves the robustness of the minimization schemes when compared with a non-adaptive choice; moreover, they show that the proposed methods could be particularly suitable to solve large scale problems where $L-B F G S$ performs poorly.
\end{abstract}

Keywords - Unconstrained minimization quasi-Newton methods matrix algebras matrix projections preserving directions

\section{Introduction}

In minimizing a function $f: \mathbb{R}^{n} \rightarrow \mathbb{R}$, in order to reduce the computational cost per iteration and the memory required for implementation of the well known BFGS minimization method, it is proposed in $[16,19,17,18,15]$ to use a $B F G S$-type updating scheme which updates, at each step, a suitable approximation of the Hessian approximation $B_{k}$, usually denoted by $\widetilde{B}_{k}$. This scheme is named $\mathcal{L} Q N$ when the matrix $\widetilde{B}_{k}$ is the projection $\mathcal{L}_{B_{k}}$ of the matrix $B_{k}$ in a matrix algebra $\mathcal{L}$ of matrices simultaneously diagonalized by a given unitary transform $U$ (we write $\mathcal{L}:=\operatorname{sd} U$, see (2) for a precise definition). The implementation of the $\mathcal{L}$ QN turns out to be very cheap when $U$ defines a low complexity transform.

While in $[4,9,24] \mathcal{L}$ is a fixed matrix algebra, in $[15,17]$ it is observed that an adaptive choice of $\mathcal{L}$, i.e, using different algebras $\mathcal{L}^{(k)}$ for each iteration $k$, could preserve more information from the original matrix $B_{k}$, and thus improve the efficiency of $\mathcal{L}$ QN. In [12] it is introduced a convergent $\mathcal{L}^{(k)} \mathrm{QN}$ scheme whose effectiveness is shown by preliminary numerical experiences.

The main contribution of this work is twofold. On the one hand we extend the theoretical framework and the convergence theory developed in $[16,12]$ for $B F G S$-type techniques to the restricted Broyden Class-type of quasi Newton methods (for the restricted Broyden Class see [8]).

On the other hand, we consider the special Broyden Class-type methods in which the update of $B_{k}$ has the form

$$
B_{k+1}=\Phi\left(\mathcal{L}_{B_{k}}^{(k)}, \mathbf{s}_{k}, \mathbf{y}_{k}, \phi\right),
$$

where $\mathbf{s}_{k}:=\mathbf{x}_{k+1}-\mathbf{x}_{k}, \mathbf{y}_{k}:=\mathbf{g}_{k+1}-\mathbf{g}_{k}\left(\mathbf{g}_{k}:=\nabla f\left(\mathbf{x}_{k}\right)\right), \mathbf{x}_{k}$ is the current guess of the minimum and the transform $U_{k}$, which diagonalizes the matrices of $\mathcal{L}^{(k)}$, is the product of few Householder reflections. Exploiting the fact that a Householder reflection is a rank one modification of the identity, we propose an algorithm to implement the update in equation (1) using $O(n)$ operations per step: hence the complexity of the Quasi-Newton methods so obtained is comparable to the more traditional methods of limited-memory type. Additionally, we show that if the projections $\mathcal{L}_{B_{k}}^{(k)}$ are such that

$$
\operatorname{tr} \mathcal{L}_{B_{k}}^{(k)} \leq \operatorname{tr} B_{k}, \quad \operatorname{det} \mathcal{L}_{B_{k}}^{(k)} \geq \operatorname{det} B_{k},
$$

\footnotetext{
*Department of Mathematics "Tullio Levi-Civita", University of Padua, Padova, Italy (stefano.cipolla@unipd.it)

†University of Rome "Tor Vergata" Via Della Ricerca Scientifica 1, 00133 Rome

(difiore@mat.uniroma2.it, zellini@mat.uniroma2.it)
} 
and

$$
\mathcal{L}_{B_{k}}^{(k)} \mathbf{s}_{k}=B_{k} \mathbf{s}_{k},
$$

then the new $\mathcal{L}^{(k)} \mathrm{QN}$ method is sound (see Algorithm 3) if the objective function is convex and has a minimizer (see Theorem 1 , Theorem 3 and Corollary 1 ).

The $\mathcal{L}^{(k)}$ QN methods so obtained turn out to be a remarkable refinement of the methods introduced in [12]. Observe that equation (II), which allows to mimic the BFGS self correction properties (see Section 4 ), is equivalent to the equality $\left(\mathcal{L}_{B_{k}}^{(k)}\right)^{-1} \mathbf{g}_{k}=B_{k}^{-1} \mathbf{g}_{k}$, i.e., the new introduced method (Algorithm 3 ) belongs to both the $\mathcal{S}$ ecant and $\mathcal{N}$ on $\mathcal{S}$ ecant class of Broyden Class-type methods (see $[16,12]$ and Section 2.2 for the precise definitions), thus rising a question on the very meaning of secant equation in Quasi-Newton methods [12]. Moreover, developing a further adaptive criterion (see (58)) for the choice of $\widetilde{B}_{k}=\mathcal{L}_{B_{k}}^{(k)}$, we produce a low complexity convergent $\mathcal{L}^{(k)} \mathrm{QN}$ with quadratic termination property (see Algorithm 5).

The proposed adaptive criteria can be satisfied by $\mathcal{L}^{(k)}=\operatorname{sd} U_{k}$ where $U_{k}$ is the product of three Householder matrices. Algorithm 3 and Algorithm 5 can be implemented by storing, respectively, 15 or 17 vectors of length $n$, whereas $L-B F G S$ - a limited memory version of $B F G S$ suitable to solve large scale problems $[22,34]$ - requires $2 M+2$ vectors of length $n$ (being $M$ the number of $\mathbf{s}_{j}, \mathbf{y}_{j}$ used to define $B_{k+1}$ ). Even if $L-B F G S$ is usually used with small values of $M$, it is well known that for some problems (see for example [28]) a greater value of $M$ could be required, and hence, for these problems, the memory required for the implementation of the algorithms here proposed could be considerably smaller. Note, moreover, that in contrast with $L-B F G S$ where some information is discarded at each step, in Algorithm 3 and Algorithm 5 the second order information generated in all the previous steps is stored in an approximate way.

Using performance profiles [21] based on iterations, function evaluations and time, the results of numerical experiences on set of problems, taken from CUTEst [26], are provided. These experiences confirm that the proposed scheme (Algorithm 5) permits to guarantee a better level of approximation of second order information if compared with $L-B F G S$ (even if a big value of $M$ is chosen) resulting on an increased robustness. Additional numerical experiences on a different set of problems, see Experiment 2, highlight the competitiveness of our proposals if compared to previous $\mathcal{L} Q N$ algorithms studied in literature.

Moreover, following the ideas developed, for instance, in [1,2], a suitable scaling improves the efficiency of $\mathcal{L}^{(k)} Q N$. In particular, Scaled $(\mathrm{Sc}) \mathcal{L}^{(k)} \mathrm{QN}$ turns out to be competitive, in some cases, with respect to $L-B F G S$ (see Remark 7 and Section 8).

\section{$2 \quad$ Notation and preliminaries}

We will freely use familiar properties of symmetric positive definite matrices and fundamental results concerning algebras of matrices simultaneously diagonalized by a given unitary transform.

We use the shorthand pd to denote a real symmetric positive definite matrix. Given a vector $\mathbf{z} \in \mathbb{R}^{n}$ we write $\mathbf{z}>0$ to denote entry-wise positivity. Let $d(\mathbf{z})$ be the diagonal matrix whose diagonal entries are the components of $\mathbf{z}$; let $d(A)$ and $\lambda(A)$ be the vectors of the diagonal entries and of the eigenvalues of a given matrix $A$, respectively. Finally, the symbol $\|\cdot\|$ will denote both the euclidean norm for vectors and the corresponding induced norm for matrices.

\subsection{Matrix Algebras}

Let $M_{n}(\mathbb{C})$ be the set of all $n \times n$ matrices with complex entries. Given a unitary matrix $U \in M_{n}(\mathbb{C})$ (i.e. $\mathrm{U} n \times n$ and $U^{H}=U^{-1}$ ), define the following algebra $\mathcal{L}$ of matrices:

$$
\mathcal{L}:=\operatorname{sd} U=\left\{U d(\mathbf{z}) U^{H}: \mathbf{z} \in \mathbb{C}^{n}\right\} .
$$

Given a matrix $B \in M_{n}(\mathbb{C})$, by the Hilbert projection theorem, there exists a unique element $\mathcal{L}_{B} \in \mathcal{L}$ such that

$$
\left\|\mathcal{L}_{B}-B\right\|_{F} \leq\|X-B\|_{F}, \quad \forall X \in \mathcal{L}
$$

where $\|\cdot\|_{F}$ denotes the Frobenius norm. It is easy to find the following explicit formula for $\mathcal{L}_{B}$ (see for example $[16]$ ):

$$
\mathcal{L}_{B}=U d\left(\mathbf{z}_{B}\right) U^{H}, \text { where }\left[\mathbf{z}_{B}\right]_{i}=\left[U^{H} B U\right]_{i i}, i=1, \ldots, n .
$$

$\mathcal{L}_{B}$ will be called the best approximation in Frobenius norm of $B$ in $\mathcal{L}$.

For the sake of completeness we recall hereafter few important results on the projection $\mathcal{L}_{B}$ of a matrix $B$ onto a sd $U$ subspace. 
Lemma 1. Let $U$ be an unitary matrix, let $\mathcal{L}=\operatorname{sd} U$ and let $B \in M_{n}(\mathbb{C})$.

1. If $B=\mathbf{x y}^{T}$, then $z_{\mathbf{x y}^{T}}=d\left(U^{H} \mathbf{x}\right) U^{T} \mathbf{y}$ where $\mathbf{x}, \mathbf{y} \in \mathbb{C}^{n}$.

2. If $B=B^{H}$, then $\mathcal{L}_{B}=\mathcal{L}_{B}^{H}$ and $\min \lambda(B) \leq \lambda\left(\mathcal{L}_{B}\right) \leq \max \lambda(B)$ where $\lambda(X)$ denotes the generic eigenvalue of $X$. Therefore $\mathcal{L}_{B}$ is Hermitian positive definite whenever $B$ is Hermitian positive definite.

3. If $B \in \mathbb{R}^{n \times n}$ then $\mathcal{L}_{B} \in \mathbb{R}^{n \times n}$ whenever $\mathcal{L}$ is closed under conjugation (i.e., $A \in \mathcal{L} \Rightarrow$ $\bar{A} \in \mathcal{L})$.

4. $\operatorname{tr}\left(\mathcal{L}_{B}\right)=\operatorname{tr}(B)$

5. If $B$ is $p d$, then $\operatorname{det}(B) \leq \operatorname{det}\left(\mathcal{L}_{B}\right)$ where the equality holds iff $U$ diagonalizes $B$, i.e., iff $U^{H} B U$ is diagonal.

Proof. For 1. see [16], for 2., 3. and 4. see Propositions 5.2 in [20]. Concerning 5., let $A$ be a pd matrix. Then we have $\operatorname{det} A \leq \prod_{i=1}^{n} a_{i i}$ (Hadamard inequality, see [27]), and $\operatorname{det}(A)=\prod_{i=1}^{n} a_{i i}$ if and only if $A$ is diagonal (see Theorem 7.8.1 [27] ). In order to obtain 5 . it is sufficient to apply these remarks to the pd matrix $U^{H} B U$. In fact, we have

$$
\operatorname{det}(B)=\operatorname{det}\left(U^{H} B U\right) \leq \prod_{i=1}^{n}\left(U^{H} B U\right)_{i i}=\operatorname{det}\left(\mathcal{L}_{B}\right)
$$

and equality holds if and only if $U^{H} B U$ is diagonal.

The properties 4 . and 5. of Lemma 1 will be crucial to state the conditions (18) and (19), for the convergence of the new method (see Theorem 1).

For a more exhaustive treatment of the contents of Lemma 1, and its relevance for $\mathcal{L}^{(k)} \mathrm{QN}$ minimizations algorithms and optimal preconditioning of linear systems, one can see [16] and [20]. Even if in the following sections we will use real unitary matrices $U$, in many situations the transform $U$ that diagonalizes matrices of $\mathcal{L}$, is defined on $\mathbb{C}$. This is the typical case of circulant matrices, where $U$ is the Fourier transform. Then, to maintain a suitable degree of generality, the notation $U^{H}$ is necessary instead of $U^{T}$, and partial results of the computational process, implicit in the iteration step $B_{k}=\Phi\left(\mathcal{L}_{B_{k}}^{(k)}, \mathbf{s}_{k}, \mathbf{y}_{k}, \phi\right)$ (see Algorithm 3), will be complex numbers. This does not compromise the fact that in each instruction the final numerical results are real. However, in this paper we will consider just real transforms $U$, so we will exchange the word 'unitary' with the word 'orthogonal' and the superscript ' $H$ ' (Hermitian) with the superscript ' $T$ ' (transpose) from the next section on.

The algebras $\mathcal{L}$ considered in this article will be of low complexity, i.e., the matrix vector product $A \mathrm{x}$, for $A \in \mathcal{L}$, will be computable in a number of operations which grows slower than $O\left(n^{2}\right)$.

\subsection{Broyden Class-type methods}

Let us consider a function $f: \mathbb{R}^{n} \rightarrow \mathbb{R}$ where $n \geq 2$.

In this paper we will study the following class of minimization methods obtained by generalizing the Broyden Class methods considered in [8]:

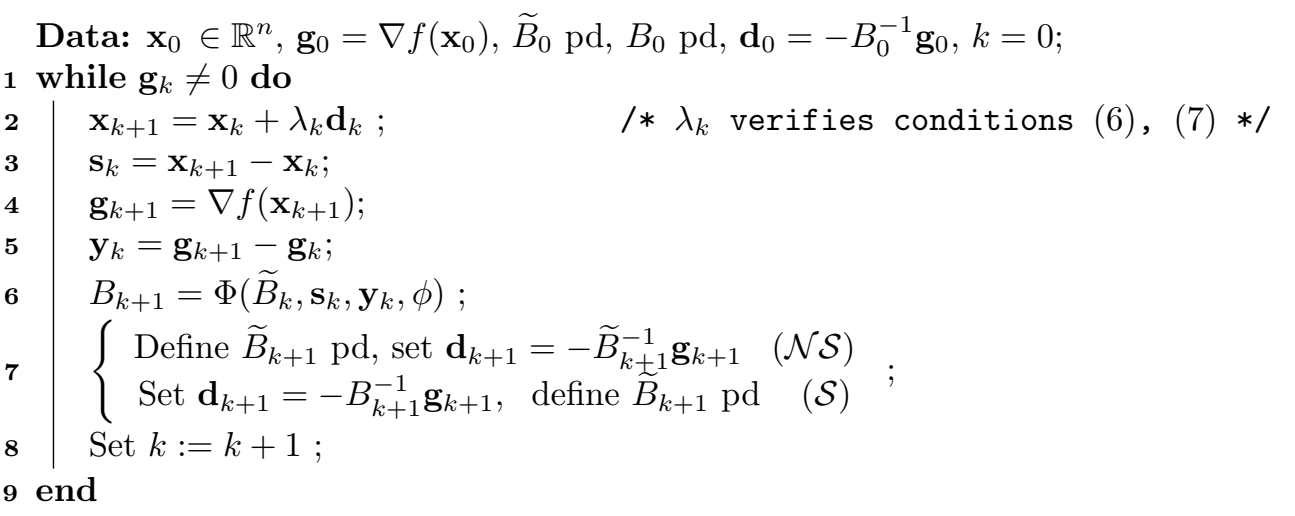

Algorithm 1: Broyden Class-type

where $\widetilde{B}_{k}$ is an approximation of $B_{k}$ and the updating formula is the Broyden's one applied to $\widetilde{B}_{k}$, i.e.

$$
\Phi\left(\widetilde{B}_{k}, \mathbf{s}_{k}, \mathbf{y}_{k}, \phi\right):=\widetilde{B}_{k}-\frac{\widetilde{B}_{k} \mathbf{s}_{k} \mathbf{s}_{k}^{T} \widetilde{B}_{k}}{\mathbf{s}_{k}^{T} \widetilde{B}_{k} \mathbf{s}_{k}}+\frac{\mathbf{y}_{k} \mathbf{y}_{k}^{T}}{\mathbf{y}_{k}^{T} \mathbf{s}_{k}}+\phi \mathbf{s}_{k}^{T} \widetilde{B}_{k} \mathbf{s}_{k} \mathbf{v}_{k} \mathbf{v}_{k}^{T}
$$


In (5) the vector $\mathbf{v}_{k}$ is defined by

$$
\mathbf{v}_{k}=\frac{\mathbf{y}_{k}}{\mathbf{y}_{k}^{T} \mathbf{s}_{k}}-\frac{\widetilde{B}_{k} \mathbf{s}_{k}}{\mathbf{s}_{k}^{T} \widetilde{B}_{k} \mathbf{s}_{k}}
$$

and $\phi$ is a non negative parameter so that $\Phi\left(\widetilde{B}_{k}, \mathbf{s}_{k}, \mathbf{y}_{k}, \phi\right)$ is pd whenever $\widetilde{B}_{k}$ is pd and $\mathbf{y}_{k}^{T} \mathbf{s}_{k}>0$.

For $\phi \in[0,1]$ we call the Broyden Class-type family "restricted". If $\widetilde{B}_{k}=B_{k}$ for all $k$, then for $\phi=0$ and $\phi=1$ one obtains, respectively, the BFGS and the DFP method [34].

We assume that the step-length parameter $\lambda_{k}$ is chosen by an inexact line search satisfying the Wolfe conditions

$$
\begin{gathered}
f\left(\mathbf{x}_{k}+\lambda_{k} \mathbf{d}_{k}\right) \leq f\left(\mathbf{x}_{k}\right)+\alpha \lambda_{k} \mathbf{g}_{k}^{T} \mathbf{d}_{k} \\
g\left(\mathbf{x}_{k}+\lambda_{k} \mathbf{d}_{k}\right)^{T} \mathbf{d}_{k} \geq \beta \mathbf{g}_{k}^{T} \mathbf{d}_{k}
\end{gathered}
$$

where $0<\alpha<1 / 2$ and $\alpha<\beta<1$. Condition (7) implies $\mathbf{y}_{k}^{T} \mathbf{s}_{k}>0$.

Let us observe that in the $\mathcal{S}$ case of Algorithm 1, the matrices generating the search directions $\mathbf{d}_{k+1}$ satisfy the Secant Equation $B_{k+1} \mathbf{s}_{k}=\mathbf{y}_{k}$. Instead, in the $\mathcal{N S}$ case such property is not necessarily fulfilled, i.e., in general, $\widetilde{B}_{k+1} \mathbf{s}_{k} \neq \mathbf{y}_{k}$.

In the following three remarks we collect some useful properties we will use in Section 3.

Remark 1. Observe that

$$
\begin{array}{r}
\operatorname{tr}\left(B_{k+1}\right)=\operatorname{tr}\left(\Phi\left(\widetilde{B}_{k}, \mathbf{s}_{k}, \mathbf{y}_{k}, \phi\right)\right)=\operatorname{tr}\left(\widetilde{B}_{k}\right)+\frac{\left\|\mathbf{y}_{k}\right\|^{2}}{\mathbf{y}_{k}^{T} \mathbf{s}_{k}}+\phi \frac{\left\|\mathbf{y}_{k}\right\|^{2}}{\mathbf{y}_{k}^{T} \mathbf{s}_{k}} \frac{\mathbf{s}_{k}^{T} \widetilde{B}_{k} \mathbf{s}_{k}}{\mathbf{y}_{k}^{T} \mathbf{s}_{k}} \\
-(1-\phi) \frac{\left\|\widetilde{B}_{k} \mathbf{s}_{k}\right\|^{2}}{\mathbf{s}_{k}^{T} \widetilde{B}_{k} \mathbf{s}_{k}}-2 \phi \frac{\mathbf{y}_{k}^{T} \widetilde{B}_{k} \mathbf{s}_{k}}{\mathbf{y}_{k}^{T} \mathbf{s}_{k}} .
\end{array}
$$

Since $\phi \mathbf{s}_{k}^{T} \widetilde{B}_{k} \mathbf{s}_{k} \geq 0$, the last term in (5) increases the eigenvalues of the previous part of the update, and hence

$$
\operatorname{det}\left(B_{k+1}\right) \geq \operatorname{det}\left(\widetilde{B}_{k}-\frac{\widetilde{B}_{k} \mathbf{s}_{k} \mathbf{s}_{k}^{T} \widetilde{B}_{k}}{\mathbf{s}_{k}^{T} \widetilde{B}_{k} \mathbf{s}_{k}}+\frac{\mathbf{y}_{k} \mathbf{y}_{k}^{T}}{\mathbf{y}_{k}^{T} \mathbf{s}_{k}}\right)=\operatorname{det}\left(\widetilde{B}_{k}\right) \frac{\mathbf{y}_{k}^{T} \mathbf{s}_{k}}{\mathbf{s}_{k}^{T} \widetilde{B}_{k} \mathbf{s}_{k}}
$$

(for the last equality see [34]).

Remark 2. From (7) it follows that, using definitions in Algorithm 1,

$$
\mathbf{y}_{k}^{T} \mathbf{s}_{k}=\mathbf{g}_{k+1}^{T} \mathbf{s}_{k}-\mathbf{g}_{k}^{T} \mathbf{s}_{k} \geq-(1-\beta) \mathbf{g}_{k}^{T} \mathbf{s}_{k}
$$

from which we obtain

$$
\frac{\mathbf{s}_{k}^{T} \widetilde{B}_{k} \mathbf{s}_{k}}{\mathbf{y}_{k}^{T} \mathbf{s}_{k}} \leq \frac{\mathbf{s}_{k}^{T} \widetilde{B}_{k} \mathbf{s}_{k}}{(1-\beta)\left(-\mathbf{g}_{k}^{T} \mathbf{s}_{k}\right)}=\frac{\lambda_{k}}{1-\beta}
$$

$\left(\mathbf{s}_{k}^{T} \widetilde{B}_{k} \mathbf{s}_{k}=\mathbf{s}_{k}^{T}\left(-\lambda_{k} \mathbf{g}_{k}\right)\right.$ in the $\mathcal{N S}$ case $)$ and

$$
\frac{\mathbf{s}_{k}^{T} B_{k} \mathbf{s}_{k}}{\mathbf{y}_{k}^{T} \mathbf{s}_{k}} \leq \frac{\mathbf{s}_{k}^{T} B_{k} \mathbf{s}_{k}}{(1-\beta)\left(-\mathbf{g}_{k}^{T} \mathbf{s}_{k}\right)}=\frac{\lambda_{k}}{1-\beta}
$$

$\left(\mathbf{s}_{k}^{T} B_{k} \mathbf{s}_{k}=\mathbf{s}_{k}^{T}\left(-\lambda_{k} \mathbf{g}_{k}\right)\right.$ in the $\mathcal{S}$ case $)$.

Remark 3. Let us define $f_{*}$ to be the infimum of $f$. Using (6) we have (in both $\mathcal{N S}$ and $\mathcal{S}$ methods)

$$
\begin{array}{r}
\sum_{k=0}^{N} \mathbf{s}_{k}^{T}\left(-\mathbf{g}_{k}\right)=\sum_{k=0}^{N}-\lambda_{k} \mathbf{d}_{k}^{T} \mathbf{g}_{k} \\
\leq \frac{1}{\alpha} \sum_{k=0}^{N}\left[f\left(\mathbf{x}_{k}\right)-f\left(\mathbf{x}_{k+1}\right)\right] \\
\leq \frac{1}{\alpha}\left[f\left(\mathbf{x}_{0}\right)-f_{*}\right]<\infty
\end{array}
$$

Then the sum converges for $n \rightarrow+\infty$, from which we obtain

$$
\lim _{k \rightarrow+\infty} \mathbf{s}_{k}^{T}\left(-\mathbf{g}_{k}\right)=0
$$




\subsection{Assumptions for the function $f$}

In Section 3, in order to obtain a convergence result for the Broyden Class-type, we will do the following:

Assumption 1. The level set

$$
D=\left\{\mathbf{x} \in \mathbb{R}^{n}: f(\mathbf{x}) \leq f\left(\mathbf{x}_{0}\right)\right\}
$$

is convex, the function $f(\mathbf{x})$ is twice continuously differentiable, convex and bounded below in $D$ and the Hessian matrix is bounded in D, i.e.

$$
\|G(\mathbf{x})\| \leq M \text {, being } M \text { a positive contant. }
$$

Remark 4. Observe that the condition (14) could be replaced by uniform convexity of $f(\mathbf{x})$ and Lipschitz condition on $G(\mathbf{x})$. Moreover, if Assumption 1 is fulfilled, then the following boundedness condition on the the Powell's ratio $\left\|\mathbf{y}_{k}\right\|^{2} / \mathbf{s}_{k}^{T} \mathbf{y}_{k}$ [37] holds:

$$
\frac{\left\|\mathbf{y}_{k}\right\|^{2}}{\mathbf{s}_{k}^{T} \mathbf{y}_{k}} \leq M
$$

where $\mathbf{s}_{k}, \mathbf{y}_{k}$ are the difference vectors produced by Algorithm 1. In fact, if we define (see [8], [34]) the pd matrix

$$
\bar{G}=\int_{0}^{1} G\left(\mathbf{x}_{k}+\tau \mathbf{s}_{k}\right) d \tau
$$

then we have from standard analysis results,

$$
\mathbf{y}_{k}=\bar{G} \mathbf{s}_{k}
$$

and hence if $\mathbf{z}_{k}=\bar{G}^{\frac{1}{2}} \mathbf{s}_{k}$,

$$
\frac{\left\|\mathbf{y}_{k}\right\|^{2}}{\mathbf{s}_{k}^{T} \mathbf{y}_{k}}=\frac{\mathbf{s}_{k}^{T} \bar{G}^{2} \mathbf{s}_{k}}{\mathbf{s}_{k}^{T} \bar{G} \mathbf{s}_{k}}=\frac{\mathbf{z}_{k}^{T} \bar{G} \mathbf{z}_{k}}{\mathbf{z}_{k}^{T} \mathbf{z}_{k}} \leq \sup _{\tau \in[0,1]}\left\|G\left(\mathbf{x}_{k}+\tau \mathbf{s}_{k}\right)\right\| \leq M .
$$

We recall that condition (15) is typically used to prove the global convergence of $B F G S$ method [37] and of $\mathcal{L} Q N$ methods [16]. Observe that, if one could impose the discrete convexity condition (15) by a suitable line-search, the convergence results in the following sections would hold under the weaker assumptions $f \in C^{1}$ and bounded below.

\section{Conditions for the convergence of the Secant and $\mathcal{N}$ on Secant Broyden Class-type}

The matrices which generate the descent directions in the $\mathcal{S}$ case exhibit explicitly second order information (or, in other words, they satisfy the secant equation). Moreover, in contrast with the limited memory versions of Quasi-Newton methods, they store, in an approximate way, the second order information generated in all the previous steps of the algorithm. In this section we will prove that both $\mathcal{S}$ and $\mathcal{N S}$ versions of Algorithm 1 are convergent if $\widetilde{B}_{k}$ is suitably chosen.

Now, using techniques and ideas developed in $[8,7]$, we state the following result which generalizes to the Broyden class of updating formulas [8] what has been proved in [12] for $B F G S$-type $\mathcal{S}$ methods.

Theorem 1. If the $\mathcal{S}$ version of Algorithm 1 with $\phi \in[0,1)$ is applied to a function that satisfies Assumption 1 and $\widetilde{B}_{k}$ is chosen such that

$$
\begin{aligned}
\operatorname{tr} \widetilde{B}_{k} & \leq \operatorname{tr} B_{k} \\
\operatorname{det} \widetilde{B}_{k} & \geq \operatorname{det} B_{k} \\
\frac{\left\|B_{k} \mathbf{s}_{k}\right\|^{2}}{\left(\mathbf{s}_{k}^{T} B_{k} \mathbf{s}_{k}\right)^{2}} & \leq \frac{\left\|\widetilde{B}_{k} \mathbf{s}_{k}\right\|^{2}}{\left(\mathbf{s}_{k}^{T} \widetilde{B}_{k} \mathbf{s}_{k}\right)^{2}} .
\end{aligned}
$$

for all $k$, then

$$
\liminf _{k \rightarrow \infty}\left\|\mathbf{g}_{k}\right\|=0
$$

for any starting point $\mathbf{x}_{0}$ and any pd matrix $B_{0}$. 
The main idea to prove Theorem 1 is to compare the third and fifth term of (8). Let us define $\psi_{k}$ as

$$
\psi_{k}:=\left[\frac{\left\|\mathbf{y}_{k}\right\|^{2}}{\mathbf{y}_{k}^{T} \mathbf{s}_{k}} \frac{\mathbf{s}_{k}^{T} \widetilde{B}_{k} \mathbf{s}_{k}}{\mathbf{y}_{k}^{T} \mathbf{s}_{k}}-2 \frac{\mathbf{y}_{k}^{T} \widetilde{B}_{k} \mathbf{s}_{k}}{\mathbf{y}_{k}^{T} \mathbf{s}_{k}}\right] \frac{\mathbf{s}_{k}^{T} \widetilde{B}_{k} \mathbf{s}_{k}}{\left\|\widetilde{B}_{k} \mathbf{s}_{k}\right\|^{2}}
$$

so that (8) becomes

$$
\operatorname{tr}\left(B_{k+1}\right)=\operatorname{tr}\left(\widetilde{B_{k}}\right)+\frac{\left\|\mathbf{y}_{k}\right\|^{2}}{\mathbf{y}_{k}^{T} \mathbf{s}_{k}}-\left(1-\phi-\psi_{k} \phi\right) \frac{\left\|\widetilde{B}_{k} \mathbf{s}_{k}\right\|^{2}}{\mathbf{s}_{k}^{T} \widetilde{B}_{k} \mathbf{s}_{k}} .
$$

In the following remarks we state upper bounds for the addends appearing in (22).

\section{Remark 5.}

$$
\begin{array}{r}
\frac{\left\|\mathbf{y}_{k}\right\|^{2}}{\mathbf{y}_{k}^{T} \mathbf{s}_{k}} \frac{\mathbf{s}_{k}^{T} \widetilde{B}_{k} \mathbf{s}_{k}}{\mathbf{y}_{k}^{T} \mathbf{s}_{k}} \frac{\mathbf{s}_{k}^{T} \widetilde{B}_{k} \mathbf{s}_{k}}{\left\|\widetilde{B}_{k} \mathbf{s}_{k}\right\|^{2}} \leq M \frac{\left(\mathbf{s}_{k}^{T} \widetilde{B}_{k} \mathbf{s}_{k}\right)^{2}}{\mathbf{y}_{k}^{T} \mathbf{s}_{k}\left\|\widetilde{B}_{k} \mathbf{s}_{k}\right\|^{2}} \\
\leq M \frac{\left(\mathbf{s}_{k}^{T} B_{k} \mathbf{s}_{k}\right)^{2}}{\mathbf{y}_{k}^{T} \mathbf{s}_{k}\left\|B_{k} \mathbf{s}_{k}\right\|^{2}}=\frac{M\left(\mathbf{s}_{k}^{T}\left(-\mathbf{g}_{k}\right)\right)^{2}}{\mathbf{y}_{k}^{T} \mathbf{s}_{k}\left\|-\mathbf{g}_{k}\right\|^{2}} \\
\leq \frac{M\left(\mathbf{s}_{k}^{T}\left(-\mathbf{g}_{k}\right)\right)}{(1-\beta)\left\|-\mathbf{g}_{k}\right\|^{2}},
\end{array}
$$

where first inequality follows using (15), the second using (20) and last inequality follows using (10).

\section{Remark 6.}

$$
\begin{aligned}
\frac{\left|\mathbf{y}_{k}^{T} \widetilde{B}_{k} \mathbf{s}_{k}\right|}{\mathbf{y}_{k}^{T} \mathbf{s}_{k}} \frac{\mathbf{s}_{k}^{T} \widetilde{B}_{k} \mathbf{s}_{k}}{\left\|\widetilde{B}_{k} \mathbf{s}_{k}\right\|^{2}} & \leq \frac{\left\|\mathbf{y}_{k}\right\| \mathbf{s}_{k}^{T} \widetilde{B}_{k} \mathbf{s}_{k}}{\mathbf{y}_{k}^{T} \mathbf{s}_{k}\left\|\widetilde{B}_{k} \mathbf{s}_{k}\right\|} \\
& \leq \frac{\sqrt{M} \mathbf{s}_{k}^{T} \widetilde{B}_{k} \mathbf{s}_{k}}{\sqrt{\mathbf{y}_{k}^{T} \mathbf{s}_{k}}\left\|\widetilde{B}_{k} \mathbf{s}_{k}\right\|} \\
\leq \frac{\sqrt{M} \mathbf{s}_{k}^{T} B_{k} \mathbf{s}_{k}}{\sqrt{\mathbf{y}_{k}^{T} \mathbf{s}_{k}}\left\|B_{k} \mathbf{s}_{k}\right\|} & =\frac{\sqrt{M}\left(\mathbf{s}_{k}^{T}\left(-\mathbf{g}_{k}\right)\right)}{\sqrt{\mathbf{y}_{k}^{T} \mathbf{s}_{k}}\left\|-\mathbf{g}_{k}\right\|} \\
& \leq \frac{\sqrt{M\left(\mathbf{s}_{k}^{T}\left(-\mathbf{g}_{k}\right)\right)}}{\sqrt{1-\beta}\left\|-\mathbf{g}_{k}\right\|}
\end{aligned}
$$

where the first inequality follows from Cauchy-Schwarz inequality, the second from (15), the third from (20), the fourth from (10).

We can now prove Theorem 1.

Proof. Arguing by contradiction, let us assume $\left\|\mathbf{g}_{k}\right\|$ bounded away from zero, i.e., there exists $\gamma>0$ such that

From Remark 3 we obtain

$$
\left\|\mathbf{g}_{k}\right\| \geq \gamma>0
$$

$$
\lim _{k \rightarrow \infty} \frac{\mathbf{s}_{k}^{T}\left(-\mathbf{g}_{k}\right)}{\left\|-\mathbf{g}_{k}\right\|^{2}}=0 .
$$

Now we show that (27) leads to a contradiction, thus (26) cannot hold. From (22), using Remark 5, Remark 6 and (27) we obtain

$$
\lim _{k \rightarrow \infty} \psi_{k}=0
$$

Using (28), since $\phi \in[0,1)$, we have that there exist an index $s$ and constants $l_{1}>0, l_{2}>0$ such that

$$
l_{2} \geq\left(1-\phi-\psi_{k} \phi\right) \geq l_{1}>0 \text { for all } k \geq s .
$$

Then we can write (for $j \geq s$ ), using (23),

$$
\operatorname{tr} B_{j+1} \leq \operatorname{tr} B_{s}+\sum_{k=s}^{j} \frac{1}{\mathbf{y}_{k}^{T} \mathbf{s}_{k}}\left\|\mathbf{y}_{k}\right\|^{2}-\sum_{k=s}^{j} \frac{1}{\mathbf{s}_{k}^{T} \widetilde{B}_{k} \mathbf{s}_{k}}\left\|\widetilde{B}_{k} \mathbf{s}_{k}\right\|^{2}\left(1-\phi-\psi_{k} \phi\right),
$$

and hence

$$
\operatorname{tr} B_{j+1} \leq \operatorname{tr} B_{s}+\sum_{k=s}^{j} \frac{1}{\mathbf{y}_{k}^{T} \mathbf{s}_{k}}\left\|\mathbf{y}_{k}\right\|^{2} \leq \operatorname{tr} B_{s}+M(j+1-s) \leq c_{1}(j+2-s)
$$


where $c_{1}=\max \left\{\operatorname{tr} B_{s}, M\right\}$ (the trace grows at most linearly for all $j \geq s$ ).

Let us remember that, given $n$ real positive numbers $a_{i}$, it holds:

$$
\prod_{i=1}^{n} a_{i} \leq\left(\frac{\sum_{i=1}^{n} a_{i}}{n}\right)^{n}
$$

from which we obtain:

$$
\operatorname{det} B_{j+1}=\prod_{i=1}^{n} \lambda_{i}\left(B_{j+1}\right) \leq\left(\frac{\sum_{i=1}^{n} \lambda_{i}\left(B_{j+1}\right)}{n}\right)^{n} \leq\left(\frac{c_{1}(j+2-s)}{n}\right)^{n} .
$$

Let us note, moreover, that from (30) and (31), since $B_{j+1}$ is pd, we have:

$$
\begin{array}{r}
\sum_{k=s}^{j} \frac{1}{\mathbf{s}_{k}^{T} \widetilde{B}_{k} \mathbf{s}_{k}}\left\|\widetilde{B}_{k} \mathbf{s}_{k}\right\|^{2}\left(1-\phi-\psi_{k} \phi\right) \leq \operatorname{tr} B_{s}-\operatorname{tr} B_{j+1}+\sum_{k=s}^{j} \frac{1}{\mathbf{y}_{k}^{T} \mathbf{s}_{k}}\left\|\mathbf{y}_{k}\right\|^{2} \\
\leq \operatorname{tr} B_{s}+\sum_{k=s}^{j} \frac{1}{\mathbf{y}_{k}^{T} \mathbf{s}_{k}}\left\|\mathbf{y}_{k}\right\|^{2} \leq c_{1}(j+2-s)
\end{array}
$$

and applying once more (32) we have:

$$
\prod_{k=s}^{j} \frac{1}{\mathbf{s}_{k}^{T} \widetilde{B}_{k} \mathbf{s}_{k}}\left\|\widetilde{B}_{k} \mathbf{s}_{k}\right\|^{2}\left(1-\phi-\psi_{k} \phi\right) \leq\left(2 c_{1}\right)^{j+1-s} .
$$

From (9) and (19) we have:

$$
\operatorname{det} B_{j+1} \geq \frac{\mathbf{s}_{j}^{T} \mathbf{y}_{j}}{\mathbf{s}_{j}^{T} \widetilde{B}_{j} \mathbf{s}_{j}} \operatorname{det} \widetilde{B}_{j} \geq \frac{\mathbf{s}_{j}^{T} \mathbf{y}_{j}}{\mathbf{s}_{j}^{T} \widetilde{B}_{j} \mathbf{s}_{j}} \operatorname{det} B_{j},
$$

from which we obtain:

$$
\prod_{k=s}^{j} \frac{\mathbf{s}_{k}^{T} \mathbf{y}_{k}}{\mathbf{s}_{k}^{T} \widetilde{B}_{k} \mathbf{s}_{k}} \leq \frac{\operatorname{det} B_{j+1}}{\operatorname{det} B_{s}}
$$

From (10) we have

$$
(1-\beta)^{j+1-s} \leq \prod_{k=s}^{j} \frac{\mathbf{s}_{k}^{T} \mathbf{y}_{k}}{-\mathbf{g}_{k}^{T} \mathbf{s}_{k}},
$$

and hence, by the equality $B_{k} \mathbf{s}_{k}=-\lambda_{k} \mathbf{g}_{k}$ and by (20), (33), (35), (36),

$$
\begin{array}{r}
(1-\beta)^{j+1-s} \prod_{k=s}^{j} \frac{\left\|\mathbf{g}_{k}\right\|^{2}}{\mathbf{s}_{k}^{T}\left(-\mathbf{g}_{k}\right)}\left(1-\phi-\psi_{k} \phi\right) \\
\leq \prod_{k=s}^{j}\left(1-\phi-\psi_{k} \phi\right) \frac{\left\|-\lambda_{k} \mathbf{g}_{k}\right\|^{2}}{\mathbf{s}_{k}^{T}\left(-\lambda_{k} \mathbf{g}_{k}\right)} \frac{\mathbf{s}_{k}^{T} \mathbf{y}_{k}}{\mathbf{s}_{k}^{T}\left(-\lambda_{k} \mathbf{g}_{k}\right)} \\
=\prod_{k=s}^{j}\left(1-\phi-\psi_{k} \phi\right) \frac{\left\|B_{k} \mathbf{s}_{k}\right\|^{2}}{\mathbf{s}_{k}^{T} B_{k} \mathbf{s}_{k}} \frac{\mathbf{s}_{k}^{T} \mathbf{y}_{k}}{\mathbf{s}_{k}^{T} B_{k} \mathbf{s}_{k}} \\
\leq \prod_{k=s}^{j}\left(1-\phi-\psi_{k} \phi\right) \frac{\left\|\widetilde{B}_{k} \mathbf{s}_{k}\right\|^{2}}{\mathbf{s}_{k}^{T} \widetilde{B}_{k} \mathbf{s}_{k}} \frac{\mathbf{s}_{k}^{T} \mathbf{y}_{k}}{\mathbf{s}_{k}^{T} \widetilde{B}_{k} \mathbf{s}_{k}} \\
\leq\left(2 c_{1}\right)^{j+1-s}\left(\frac{c_{1}(j+2-s)}{n}\right)^{n} \frac{1}{\operatorname{det} B_{s}},
\end{array}
$$

i.e.,

$$
\prod_{k=s}^{j}\left(1-\phi-\psi_{k} \phi\right) \frac{\left\|\mathbf{g}_{k}\right\|^{2}}{\mathbf{s}_{k}^{T}\left(-\mathbf{g}_{k}\right)} \leq c_{2}^{j+1-s} \text { for all } j \geq s,
$$

for a suitable constant $c_{2}$ dependent on $s$ and $M$ (defined in Assumption 1). For the details see Appendix 2.

On the other hand, by (27) and by the bound $1-\phi-\psi_{k} \phi \geq l_{1}>0$ in (29), we have that the ratios $\left(1-\phi-\psi_{k} \phi\right)\left\|\mathbf{g}_{k}\right\|^{2} / \mathbf{s}_{k}^{T}\left(-\mathbf{g}_{k}\right)$ go to $+\infty$, as $k \rightarrow+\infty$; thus a natural number $j^{*} \geq s$ must exist such that

$$
\prod_{k=s}^{j}\left(1-\phi-\psi_{k} \phi\right) \mathbf{f}\left\|\mathbf{g}_{k}\right\|^{2} \mathbf{s}_{k}^{T}\left(-\mathbf{g}_{k}\right)>c_{2}^{j+1-s}, \quad \forall j \geq j^{*},
$$

(see again Appendix 2 for more details) but this contradicts (38) choosing $j \geq \max \left\{s, j^{*}\right\}$. We have hence proved that (21) holds. 
The condition (20) is satisfied, in particular, when $\widetilde{B}_{k}$ is such that

$$
\widetilde{B}_{k} \mathbf{s}_{k}=B_{k} \mathbf{s}_{k} .
$$

In the following the above equality has a crucial role. As it is clear from Algorithm 1, the equality (39) regards the basic relationship between the search directions produced by $\mathcal{S}$ and $\mathcal{N S}$ algorithms. In fact, if equality (39) holds, such search directions are perfectly equivalent even if $B_{k} \neq \widetilde{B}_{k}$. To prove the convergence property of the $\mathcal{S}$ scheme we have exploited the condition (20), which is fulfilled if (39) is fulfilled.

In the next Sections 4 and 5 we will investigate some further consequences of condition (39) and we will prove that it can be imposed by choosing $\widetilde{B}_{k}$ as the projection of $B_{k}$ onto algebras of matrices diagonalized by a fixed, small number of orthogonal Householder transforms.

The following result generalizes what proven in [16] for BFGS-type $\mathcal{N S}$ methods.

Theorem 2. If the $\mathcal{N S}$ version of Algorithm 1 with $\phi \in[0,1)$ is applied to a function that satisfies Assumption 1 and $\widetilde{B}_{k}$ is chosen such that (18) and (19) hold for all $k$, then

$$
\liminf _{k \rightarrow \infty}\left\|\mathbf{g}_{k}\right\|=0
$$

for any starting point $\mathbf{x}_{0}$ and any pd matrix $B_{0}$.

Proof. Proceed as in the proof of Theorem 1 noting that the hypothesis (20) on $\widetilde{B}_{k}$ is no longer necessary to obtain Remark 5 (see (24)), Remark 6 (see (25)) and (37), since in $\mathcal{N S}$ methods $\widetilde{B}_{k} \mathbf{s}_{k}$ turns out to be equal to $-\lambda_{k} \mathbf{g}_{k}$.

In Figure 1 we illustrate in a pictorial way the restricted Broyden Class-type $\mathcal{S}$ ecant and $\mathcal{N}$ on Secant methods satisfying the conditions $\operatorname{tr} \widetilde{B}_{k} \leq \operatorname{tr} B_{k}$, det $\widetilde{B}_{k} \geq \operatorname{det} B_{k}$ and $f \in C^{2}$, which appear basic in proving convergence results for both classes of methods. At the moment only a subset of the pictured $\mathcal{S}$ ecant methods are certainly convergent, those satisfying the surplus condition (20). In the following we will focus on Broyden Class-type methods such that $\widetilde{B}_{k} \mathbf{s}_{k}=B_{k} \mathbf{s}_{k}$, which form a subset of the intersection between convergent $\mathcal{S}$ and $\mathcal{N S}$, with the aim to define new efficient BFGS-type algorithms.

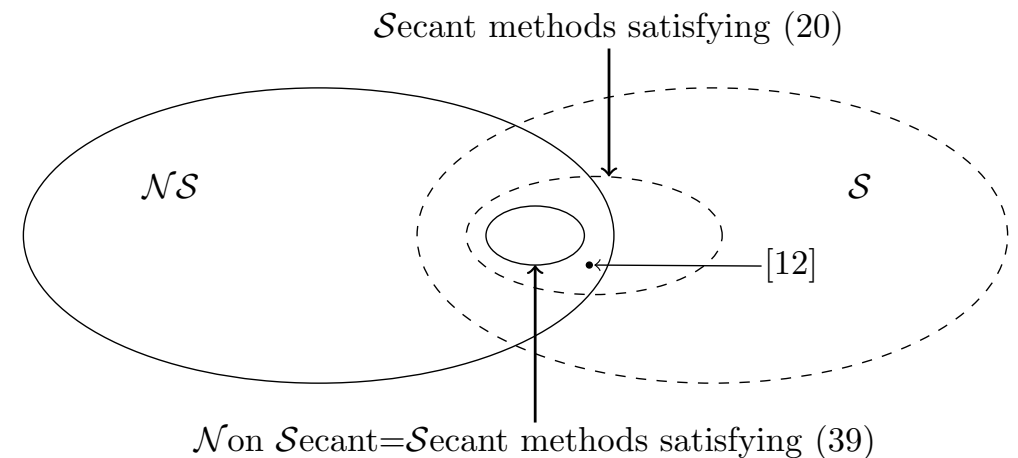

Figure 1: Restricted Broyden Class-type methods satisfying the conditions on trace, determinant.

\section{Self correcting properties implied by convergence con- ditions}

In this section, assuming $\phi=0$ in Algorithm 1, we will study how (39) reverberates on self correcting properties of the algorithm.

There are experimental evidences (in the case the matrix $\widetilde{B}_{k}$ is chosen in some fixed matrix algebra $\mathcal{L}$ ), that the $\mathcal{S}$ version of Algorithm 1 performs better if compared with the $\mathcal{N S}$ one (see [4] and [9]). In this section we will try to motivate theoretically this experimental observation by comparing $\operatorname{tr} B_{k+1}$ and $\operatorname{det} B_{k+1}$ produced by classic $B F G S$ and Algorithm 1 when $\phi=0$. Observe moreover, that in [12] some preliminary experimental experiences have shown that even if condition (39) is imposed in an approximate way (i.e $\widetilde{B}_{k} \mathbf{s}_{k} \approx B_{k} \mathbf{s}_{k}$ ) performances of Algorithm 1 are competitive with those of $\mathcal{H} Q N$, which, in turn, has been proved to be competitive with $L-B F G S$ on some neural networks problem (see $[16,4])$.

Finally let us stress the fact that, even if "the Quasi-Newton updating is inherently an overwriting process rather than an averaging process" (see [6]), the following analysis will 
show how algorithms proposed in this work exhibit an interaction between averaging and overwriting phases more similar to $B F G S$ than to $L-B F G S$ (remember that the curvature information constructed by $B F G S$ are good enough to endow the algorithm with a superlinear rate of convergence, see [34]).

Performing one step of the "classic" BFGS, one has (see (8) and (9))

$$
\begin{gathered}
B_{k+1}=\Phi\left(B_{k}, \mathbf{s}_{k}, \mathbf{y}_{k}, 0\right) \\
\operatorname{tr} B_{k+1}=\operatorname{tr} B_{k}-\frac{\left\|B_{k} \mathbf{s}_{k}\right\|^{2}}{\mathbf{s}_{k}^{T} B_{k} \mathbf{s}_{k}}+\frac{\left\|\mathbf{y}_{k}\right\|^{2}}{\mathbf{y}_{k}^{T} \mathbf{s}_{k}} \\
\operatorname{det}\left(B_{k+1}\right)=\operatorname{det}\left(B_{k}\right) \frac{\mathbf{y}_{k}^{T} \mathbf{s}_{k}}{\mathbf{s}_{k}^{T} B_{k} \mathbf{s}_{k}}=\operatorname{det}\left(B_{k}\right) \frac{\mathbf{s}_{k}^{T}\left(\bar{G} \mathbf{s}_{k}\right)}{\mathbf{s}_{k}^{T} B_{k} \mathbf{s}_{k}},
\end{gathered}
$$

from which it is possible to observe that BFGS (and all updates in the restricted Broyden class) "have a strong self correcting property with respect to the determinant" (see [8]). In particular curvatures of the model are inflated or deflated (and hence corrected) accordingly to the ratio $\frac{\mathbf{s}_{k}^{T}\left(\bar{G} \mathbf{s}_{k}\right)}{\mathbf{s}_{k}^{T} B_{k} \mathbf{s}_{k}}$, allowing the algorithm to compare the computed model with the true Hessian. In fact, the previous ratio is used to correct the spectrum of the operator defining the descent direction at next step.

On the contrary, by performing one step of Algorithm 1, we obtain equations (41) and (42) where $B_{k}$ is replaced by $\widetilde{B}_{k}$. It is then clear that if $\widetilde{B}_{k} \mathbf{s}_{k}$ is not suitably chosen, then the ratio $\frac{\mathbf{s}_{k}^{T}\left(\overline{\mathbf{s}_{k}}\right)}{\mathbf{s}_{k}^{T} \widetilde{B}_{k} \mathbf{s}_{k}}$ could not exhibit a reasonable behavior, making the algorithm not able to self-correct bad estimated curvatures and hence loosing efficiency. Hypothesis (39) is hence further justified from the "self-correcting properties point of view". Observe that if we choose $\widetilde{B}_{k}=\mathcal{L}_{B_{k}}^{(k)}$, the error we introduce contributes to inappropriately inflate the curvatures of the model because by Lemma 1 , even if $\operatorname{tr} \widetilde{B}_{k}=\operatorname{tr} B_{k}$, we have $\operatorname{det} \widetilde{B}_{k} \geq \operatorname{det} B_{k}$ (see [32] and references therein for more information regarding the inappropriate inflations problems affecting $B F G S$ ). Recall that by the same Lemma 1 , det $\widetilde{B}_{k}=\operatorname{det} B_{k}$ iff $U_{k}$ diagonalizes $B_{k}$. Thus, in order to reduce the inappropriate inflation of the curvatures of the model, $U_{k}$ should be chosen, in principle, besides of low complexity, as close as possible to a matrix which diagonalizes $B_{k}$.

The problem concerning the possibility to exploit $\widetilde{B}_{k}$ in order to improve such self correcting properties as much as possible remains open. Anyway, the relative weakness of the hypothesis of Theorem 1 leaves room, in principle, for possible different choices of $\widetilde{B}_{k}$, besides the specific choice considered in this work, which could improve self correcting property.

A quite natural choice of $\widetilde{B}_{k}$, alternative to $\widetilde{B}_{k}=\mathcal{L}_{B_{k}}^{(k)}$, can be $\widetilde{B}_{k}=\sigma_{k} \mathcal{L}_{B_{k}}^{(k)}$ for a suitable $\sigma_{k}$, as considered in the following Remark 7 (see also [1, 2]).

Remark 7. In section 8, in order to mitigate the inappropriate inflation of the curvatures introduced by the projection operation, following a well known line of research [1, 2, 35, 36, 3], we numerically investigate the introduction of a self-scaling factor $\sigma_{k}$, i.e., we will use $\widetilde{B}_{k}=\sigma_{k} \mathcal{L}_{B_{k}}^{(k)}$. More in detail, after the construction of the matrix algebra $\mathcal{L}^{(k)}$ such that $\mathcal{L}_{B_{k}}^{(k)} \mathbf{s}_{k}=B_{k} \mathbf{s}_{k}$ (see Section 5, Line 10 of Algorithm 3 and Line 12 of Algorithm 5), we scale $\mathcal{L}_{B_{k}}^{(k)}$; in particular, we use the updating formula

$$
B_{k+1}=\Phi\left(\sigma_{k} \mathcal{L}_{B_{k}}^{(k)}, \mathbf{s}_{k}, \mathbf{y}_{k}, 0\right)
$$

where

$$
\sigma_{k}:=\max \left\{\min \left\{\frac{\mathbf{y}_{k}^{T} \mathbf{s}_{k}}{\mathbf{s}_{k}^{T} \mathcal{L}_{B_{k}}^{(k)} \mathbf{s}_{k}}, 1\right\},\left(\operatorname{det}\left(B_{k}\right) / \operatorname{det}\left(\mathcal{L}_{B_{k}}^{(k)}\right)\right)^{1 / n}\right\} .
$$

Such choice of $\sigma_{k}$ guarantees that all the hypothesis of Theorem 1 are satisfied. Moreover, as $\sigma_{k} \leq 1$ for all $k$, we have

$$
\operatorname{det}\left(B_{k+1}\right)=\operatorname{det}\left(\sigma_{k} \mathcal{L}_{B_{k}}^{(k)}\right) \frac{\mathbf{y}_{k}^{T} \mathbf{s}_{k}}{\mathbf{s}_{k}^{T} \sigma_{k} \mathcal{L}_{B_{k}}^{(k)} \mathbf{s}_{k}} \leq \operatorname{det}\left(\mathcal{L}_{B_{k}}^{(k)}\right) \frac{\mathbf{y}_{k}^{T} \mathbf{s}_{k}}{\mathbf{s}_{k}^{T} \mathcal{L}_{B_{k}}^{(k)} \mathbf{s}_{k}},
$$

which implies

$$
\operatorname{det}\left(B_{k+1}\right)=\operatorname{det}\left(\Phi\left(\sigma_{k} \mathcal{L}_{B_{k}}^{(k)}, \mathbf{s}_{k}, \mathbf{y}_{k}, 0\right)\right) \leq \operatorname{det}\left(\Phi\left(\mathcal{L}_{B_{k}}^{(k)}, \mathbf{s}_{k}, \mathbf{y}_{k}, 0\right)\right),
$$

i.e., the determinants of the matrices $B_{k+1}$ generated with the $\sigma_{k}$-scaled updating formula (43) are smaller than the determinants of the matrices $B_{k+1}$ generated through the not scaled formula (1) with $\phi=0$. 
In the experiments considered in Section 8, the choice $\widetilde{B}_{k}=\sigma_{k} \mathcal{L}_{B_{k}}^{(k)}$ turns out to improve in certain cases, the not-scaled $\mathcal{L}^{(k)} Q N$ methods and indicates a possible optimization strategy, based on $\mathcal{L}^{(k)} Q N$, competitive with $L-B F G S$.

Finally, let us observe that in [12] the authors investigated BFGS-type methods where $\sigma_{k} \mathcal{L}_{B_{k}}^{(k)} \mathbf{s}_{k}=B_{k} \mathbf{s}_{k}$ for some $\sigma_{k}>0$. Nevertheless, in [12] $\sigma_{k}$ was a parameter used in the construction of the matrix algebra $\mathcal{L}^{(k)}$.

\section{How to ensure Secant convergence conditions by low complexity matrices}

In this section we will show that it is always possible to satisfy hypothesis of Theorem 1 by a low complexity matrix $\widetilde{B}_{k}$. In particular, a matrix $\widetilde{B}_{k}$ satisfying (18), (19) and (39) will be explicitly constructed.

As noticed in Lemma 1, spectral conditions (18), (19) are always fulfilled when we choose

$$
\widetilde{B}_{k}=\mathcal{L}_{B_{k}} \text { for some } \mathcal{L}=\operatorname{sd} U .
$$

Nevertheless, the condition

$$
\mathcal{L}_{B_{k}} \mathbf{s}_{k}=B_{k} \mathbf{s}_{k}
$$

is not satisfied for a generic matrix algebra $\mathcal{L}$ and we have to face the following Problem 1 (see [12] for an analogous problem involving a parameter $\sigma$ ):

Problem 1. Given a pd matrix $A \in \mathbb{R}^{n \times n}$ and a vector $\mathbf{s} \in \mathbb{R}^{n}$, find a low complexity orthogonal matrix $U$ such that

$$
\mathcal{L}_{A} s=A s
$$

where $\mathcal{L}=$ sdU.

Observe that Problem 1 has been solved in [11] in the particular case when $\mathbf{s}$ is an eigenvector of $A$ with the aim to speed-up the Pagerank computation by the preconditioned EulerRichardson method. The following Lemma 2 completely characterizes solution of Problem 1 in this case.

Lemma 2. Le $A$ be a $n \times n$ symmetric matrix, if $\mathbf{s}$ is such that $A \mathbf{s}=\gamma \mathbf{s}$, then for any orthogonal matrix $L$ such that $\mathbf{s} /\|\mathbf{s}\|$ is among its columns, we have

$$
\mathcal{L}_{A} s=A s
$$

where $\mathcal{L}=s d L$. In particular $L$ can be chosen as an orthogonal Householder matrix.

Proof. Consider an orthogonal $L$ such that $L \mathbf{e}_{k}=\mathbf{s} /\|\mathbf{s}\|$ for some fixed $k \in\{1, \ldots, n\}$. From (4) we have $\mathcal{L}_{A}=L d\left(\mathbf{z}_{A}\right) L^{T}$ being $\mathbf{z}_{A}$ the vector

$$
\mathbf{z}_{A}=\left[\ldots,\left(L^{T} A L\right)_{i i}, \ldots\right]^{T},
$$

and hence

$$
\mathcal{L}_{A} \mathbf{s}=\left(\mathbf{z}_{A}\right)_{k} \mathbf{s}=\frac{\mathbf{s}^{T} A \mathbf{s}}{\|\mathbf{s}\|^{2}} \mathbf{s}=\gamma \mathbf{s}=A \mathbf{s} .
$$

For the second part see Lemma 5 in the Appendix.

The following Theorem 3 solves Problem 1 in the general case and, at the same time, sheds light on algorithmic details necessary for the construction of the solution. In [13] it is solved a more general problem where the projection $\mathcal{L}_{A}$ retains the action of $A$ on a set of vectors instead on a single one.

Let us begin recalling the well-known Arnoldi algorithm [39] for finding an orthogonal basis of the Krylov subspace

$$
\mathcal{K}_{m}(A, \mathbf{v}):=<\mathbf{v}, A \mathbf{v}, \ldots, A^{m-1} \mathbf{v}>.
$$

In what follows we will assume $\operatorname{dim} \mathcal{K}_{m}(A, \mathbf{v})=m$.

Data: $A, \mathbf{v}_{1}:=\mathbf{v} /\|\mathbf{v}\|_{2}$;

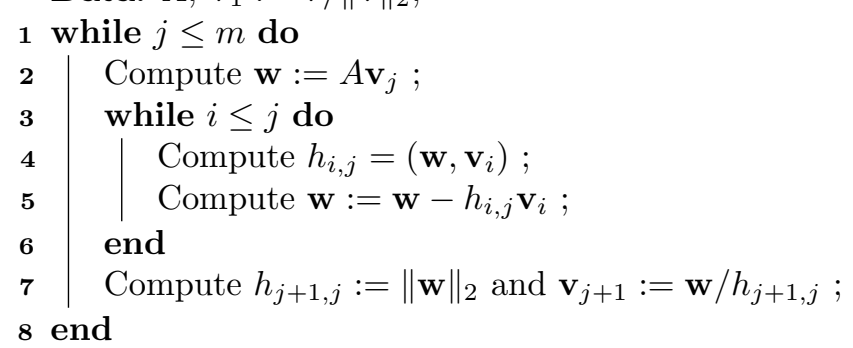

Algorithm 2: Arnoldi Algorithm 
The above algorithm produces an orthonormal basis $V_{m}=\left[\mathbf{v}_{1}, \ldots, \mathbf{v}_{m}\right]$ of the Krylov subspace $K_{m}(A, \mathbf{v})$ such that

$$
A V_{m}=V_{m} H_{m}+h_{m+1, m} \mathbf{v}_{m+1} \mathbf{e}_{m}^{T},
$$

where the matrix $H_{m}$ denotes the $m \times m$ upper Hessenberg matrix whose coefficients are the $h_{i, j}$ computed by the algorithm. From the above observations we obtain

$$
V_{m}^{T} A V_{m}=H_{m} .
$$

Moreover, the following lemma holds :

Lemma 3 ([38]). Let $A$ be $a n \times n$ real matrix and $V_{m}, H_{m}$ the results of $m$ steps of the Arnoldi or Lanczos method applied to $A$. Then for any polynomial $p_{j}$ of degree $j \leq m-1$ the following equality holds:

$$
p_{j}(A) \mathbf{v}_{1}=V_{m} p_{j}\left(H_{m}\right) \mathbf{e}_{1} .
$$

Theorem 3. Let $A \in \mathbb{R}^{n \times n}$ be a symmetric matrix. For every fixed integer $m$ and $1 \leq m \leq$ $n$ and for any $\mathbf{s} \in \mathbb{R}^{n}$ there exists an orthogonal matrix $L \in \mathbb{R}^{n \times n}$ such that if $\mathcal{L}=$ sd $L$ and $\mathcal{L}_{A}$ is the best approximation in Frobenius norm of $A$ in $\mathcal{L}$, then

$$
p_{j}\left(\mathcal{L}_{A}\right) \mathbf{s}=p_{j}(A) \mathbf{s}
$$

for any polynomial $p_{j}$ of degree $j \leq m-1$. Moreover, the thesis is satisfied also by any other orthogonal matrix having, among its columns, $m$ particular columns of L (see (52)).

Proof. Consider the matrices $V_{m}$ and $H_{m}$ constructed from Arnoldi Algorithm applied to $\mathcal{K}_{m}(A, \mathbf{s})$ (observe that the first column of $V_{m}$ is $\left.\mathbf{v}_{1}:=\mathbf{s} /\|\mathbf{s}\|\right)$. From Lemma 3 with $j=1$ we have

$$
A \mathbf{v}_{1}=V_{m} H_{m} V_{m}^{T} \mathbf{v}_{1}
$$

From (47) we can write

$$
A \mathbf{v}_{1}=V_{m} Q Q^{T} V_{m}^{T} A V_{m} Q Q^{T} V_{m}^{T} \mathbf{v}_{1}
$$

for any orthogonal matrix $Q$. In particular, being $V_{m}^{T} A V_{m}$ symmetric, we can choose in (50) $Q$ as the orthogonal matrix which diagonalizes $V_{m}^{T} A V_{m}$, i.e.

$$
A \mathbf{v}_{1}=V_{m} Q\left[\begin{array}{cccc}
x_{1} & 0 & \ldots & 0 \\
0 & \ddots & \ddots & 0 \\
0 & \ddots & \ddots & 0 \\
0 & \ldots & 0 & x_{m}
\end{array}\right] Q^{T} V_{m}^{T} \mathbf{v}_{1}
$$

where $x_{i}=\mathbf{e}_{i}^{T} Q^{T} V_{m}^{T} A V_{m} Q \mathbf{e}_{i}$ for $i=1, \ldots, m$. Consider now the matrix

$$
L=\left[V_{m} Q \mathbf{e}_{1}|\ldots| V_{m} Q \mathbf{e}_{m}\left|\mathbf{q}_{m+1}\right| \ldots \mid \mathbf{q}_{n}\right]
$$

where $\left\{\mathbf{q}_{m+1}, \ldots, \mathbf{q}_{n}\right\}$ is any orthonormal basis for

$$
<V_{m} Q \mathbf{e}_{1}, \ldots, V_{m} Q \mathbf{e}_{m}>^{\perp}=<V_{m} \mathbf{e}_{1}, \ldots, V_{m} \mathbf{e}_{m}>^{\perp}
$$

(for example $L$ can be obtained as the product of $m$ Householder matrices, see Lemma 5 in the Appendix), set $\mathcal{L}=\operatorname{sd} L$ and consider $\mathcal{L}_{A}$ the best approximation in Frobenius norm of $A$ in $\mathcal{L}$.

In order to prove that $\mathcal{L}_{A}$ satisfies (49) it is sufficient to prove that

$$
\mathcal{L}_{A}^{j} \mathbf{v}_{1}=A^{j} \mathbf{v}_{1} \text { for } 0 \leq j \leq m-1 .
$$

Of course, (54) is true for $j=0$. The equality $\mathcal{L}_{A} \mathbf{v}_{1}=A \mathbf{v}_{1}$ follows observing that using (4) we have

$$
\begin{aligned}
& \mathcal{L}_{A} \mathbf{v}_{1}=\left(\sum_{i}^{n}\left(L^{T} A L\right)_{i i} L \mathbf{e}_{i}\left(L \mathbf{e}_{i}\right)^{T}\right) \mathbf{v}_{1} \\
& =\left(\sum_{i}^{m} x_{i}\left(V_{m} Q \mathbf{e}_{i}\right)\left(V_{m} Q \mathbf{e}_{i}\right)^{T}\right) \mathbf{v}_{1}=A \mathbf{v}_{1}
\end{aligned}
$$

where in the second equality we take into account that $\mathbf{q}_{i}^{T} \mathbf{v}_{1}=0$ for $i \in\{m+1, \ldots, n\}$ (see (53)) and (52). 
Suppose now (54) true for all indexes $j$ in $[1, k], k \leq m-2$ and let us prove it for $j=k+1$. From inductive hypothesis and Lemma 3 we have

$$
\mathcal{L}_{A}^{k+1} \mathbf{v}_{1}=\mathcal{L}_{A} \mathcal{L}_{A}^{k} \mathbf{v}_{1}=\mathcal{L}_{A} A^{k} \mathbf{v}_{1}=\mathcal{L}_{A} V_{m} H_{m}^{k} \mathbf{e}_{1}
$$

From direct computation using (53) and the definition of $Q$, we have $\mathcal{L}_{A} V_{m}=V_{m} H_{m}$ and thus

$$
\mathcal{L}_{A} V_{m} H_{m}^{k} \mathbf{e}_{1}=V_{m} H_{m}^{k+1} \mathbf{e}_{1}=A^{k+1} \mathbf{v}_{1},
$$

where the last equality follows using again Lemma 3 . Hence (54) holds also for $j \in[1, k+1]$.

Corollary 1. Solutions $U$ of Problem 1 are obtained by using Theorem 3 for $m=2$ and $j=1$. Observe that just two of the columns of such orthogonal matrices $U$ are uniquely determined (they are suitable linear combinations of the vectors $\mathbf{s}$ and As), and hence one of such $U$ can be chosen as the product of two Householder matrices that can be determined by performing two products of $A$ by a vector plus $O(n)$ FLOPs.

Proof. For the second statement see (52) in the proof of Theorem 3 and Lemma 5 in the Appendix.

\subsection{Convergent $\mathcal{L}^{(k)} Q N$ scheme}

In order to impose (44) for each $k$, an adaptive choice of the space $\mathcal{L}=\operatorname{sd} U$ is necessary. Any method obtained in this way will be called $\mathcal{L}^{(k)} Q N$ extending the notation $\mathcal{L} Q N$ introduced in [16] to denote the BFGS-type methods with $\widetilde{B}_{k}=\mathcal{L}_{B_{k}}$ being $\mathcal{L}$ fixed. As a result of what discussed in Section 3 and in the first part of this section we report here the following Algorithm 3 which can be considered a refinement and an extension of the scheme proposed in [12]:

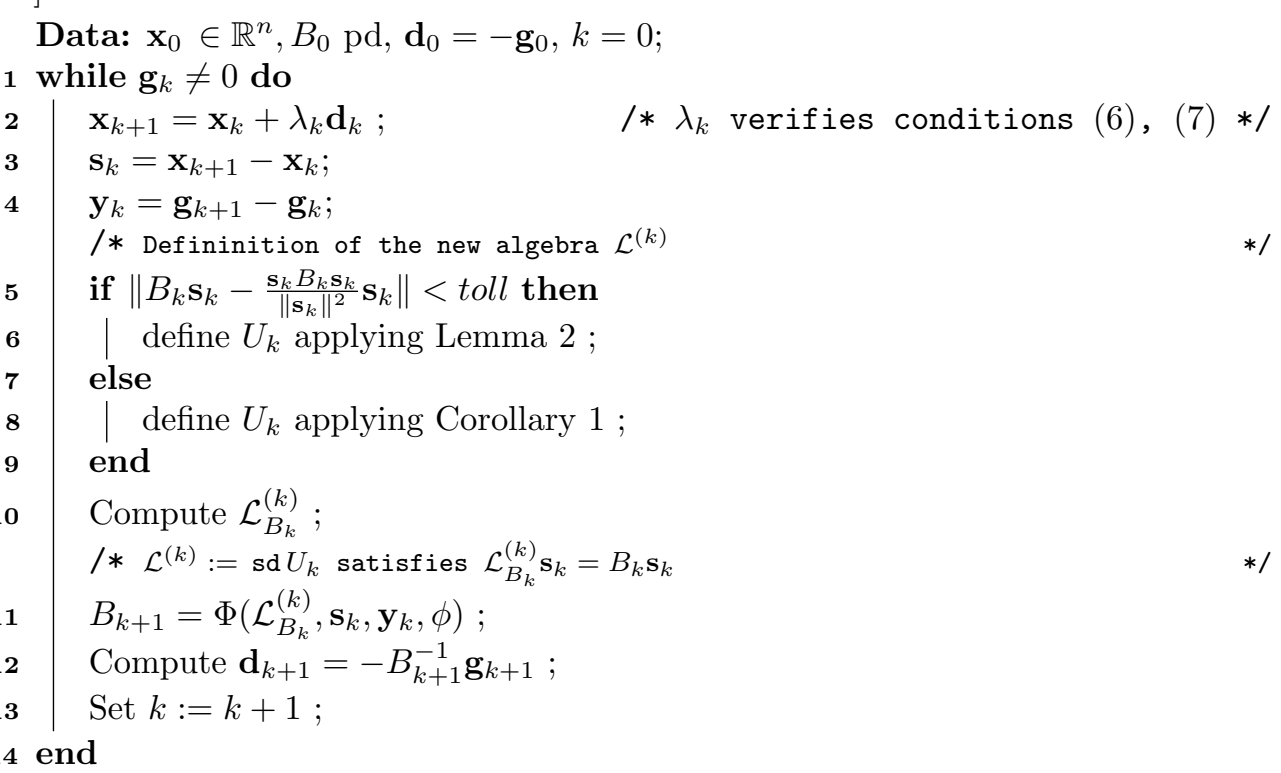

Algorithm 3: A convergent $\mathcal{L}^{(k)} \mathrm{QN}$

In more details, observe that, to perform Line 8 of Algorithm 3, it is necessary to apply Corollary 1 to $B_{k}=\Phi\left(\mathcal{L}_{B_{k-1}}^{(k-1)}, \mathbf{s}_{k-1}, \mathbf{y}_{k-1}, \phi\right)$ and $\mathbf{s}_{k}$, obtaining $U_{k}:=\mathcal{H}\left(\mathbf{h}_{2}^{(k)}\right) \mathcal{H}\left(\mathbf{h}_{1}^{(k)}\right)$. The vectors $\mathbf{h}_{1}^{(k)}$ and $\mathbf{h}_{2}^{(k)}$ can be determined by performing two products of $B_{k}$ by a vector. As $B_{k}$ is a low rank correction of the low complexity matrix $\mathcal{L}_{B_{k-1}}^{(k-1)}$, such products can be calculated in $O(n)$ FLOPs (see Corollary 1 ). To compute $\mathcal{L}_{B_{k}}^{(k)}$ in Line 10, observe that, by Lemma 1

$$
\mathcal{L}_{B_{k}}^{(k)}=\mathcal{L}_{\mathcal{L}_{B_{k-1}}^{(k-1)}}^{(k)}-\mathcal{L}_{\frac{\mathcal{L}_{B_{k-1}}^{(k-1)} \mathbf{s}_{k-1} \mathbf{s}_{k-1}^{T} \mathcal{L}_{B_{k-1}}^{(k-1)}}{(k)}}^{\left(\mathbf{s}_{k-1}^{T} \mathcal{L}_{B_{k-1}}^{(k-1)} \mathbf{s}_{k-1}\right.}+\mathcal{L}_{\frac{\mathbf{y}_{k-1} \mathbf{y}_{k-1}^{T}}{(k)}}^{\left(\mathbf{y}_{k-1}^{T} \mathbf{s}_{k-1}\right.}+\left(\phi \mathbf{s}_{k-1}^{T} \mathcal{L}_{B_{k-1}}^{(k-1)} \mathbf{s}_{k-1}\right) \mathcal{L}_{\mathbf{v}_{k-1} \mathbf{v}_{k-1}^{T}}^{(k)},
$$

and hence, it is sufficient to compute its eigenvalues (see (4)), i.e.,

$$
\begin{aligned}
\lambda\left(\mathcal{L}_{B_{k}}^{(k)}\right) & =d\left(\left[U_{k}^{T} B_{k} U_{k}\right]\right) \\
& =d\left(U_{k}^{T} \mathcal{L}_{B_{k-1}}^{(k-1)} U_{k}\right)-d\left(U_{k}^{T} \frac{\mathcal{L}_{B_{k-1}}^{(k-1)} \mathbf{s}_{k-1} \mathbf{s}_{k-1}^{T} \mathcal{L}_{B_{k-1}}^{(k-1)}}{\mathbf{s}_{k-1}^{T} \mathcal{L}_{B_{k-1}}^{(k-1)} \mathbf{s}_{k-1}} U_{k}\right)+ \\
& +d\left(U_{k}^{T} \frac{\mathbf{y}_{k-1} \mathbf{y}_{k-1}^{T}}{\mathbf{y}_{k-1}^{T} \mathbf{s}_{k-1}} U_{k}+\left(\phi \mathbf{s}_{k-1}^{T} \mathcal{L}_{B_{k-1}}^{(k-1)} \mathbf{s}_{k-1}\right) U_{k}^{T} \mathbf{v}_{k-1} \mathbf{v}_{k-1}^{T} U_{k}\right)
\end{aligned}
$$


Notice that the above equality is an extension of an eigenvalues updating formula obtained in [16] where $\mathcal{L}^{(k)} \equiv \mathcal{L}$ for all $k$

\subsection{Complexity}

For every $k$ the orthogonal matrices at Line 6 or Line 8 of Algorithm 3 are the product of at most two (only one if Line 6) Householder reflections, that can be constructed in $O(n)$ FLOPs (see Lemma 5 in the Appendix). Now, to calculate $\lambda\left(\mathcal{L}_{B_{k}}^{(k)}\right)$ in $(56)$, we compute the matrix vector products $\mathcal{L}_{B_{k-1}}^{(k-1)} \mathbf{s}_{k-1}$ in $O(n)$ FLOPs, and the same amount of operations is sufficient to compute $d\left(U_{k}^{T} \mathcal{L}_{B_{k-1}}^{(k-1)} U_{k}\right.$ ) (using Proposition 1 in [13]). Finally, observe that Line 12 of Algorithm 3 can be performed using Sherman-Morrison formula, which states that $B_{k+1}^{-1}$ is a low rank correction of $\left(\mathcal{L}_{B_{k}}^{(k)}\right)^{-1}$; for example if $\phi=0$ in Line 11 of Algorithm 3 , then

$$
B_{k+1}^{-1}=\left(I-\frac{\mathbf{s}_{k} \mathbf{y}_{k}^{T}}{\mathbf{s}_{k}^{T} \mathbf{y}_{k}}\right)\left(\mathcal{L}_{B_{k}}^{(k)}\right)^{-1}\left(I-\frac{\mathbf{y}_{k} \mathbf{s}_{k}^{T}}{\mathbf{s}_{k}^{T} \mathbf{y}_{k}}\right)+\frac{\mathbf{s}_{k} \mathbf{s}_{k}^{T}}{\mathbf{s}_{k}^{T} \mathbf{y}_{k}} .
$$

Thus it is possible to infer that the computational complexity of Algorithm 3 is $O(n)$ in space and time (to store the matrices $\mathcal{L}_{B_{k}}^{(k)}=U_{k} d\left(\mathbf{z}_{B_{k}}\right) U_{k}^{T}$ it is sufficient to store $\mathbf{z}_{B_{k}}$ and the vectors $\mathbf{h}_{i}^{(k)}$ needed to define $\left.U_{k}\right)$. When $\phi=0$, assuming that the matrices $U_{k}$ are always constructed according to Line 8 of Algorithm 3, a straightforward implementation of Algorithm 3 requires roughly $70 n$ multiplications and the storage of 15 vectors of length $n$.

\section{The quadratic finite termination property}

In literature Quasi-Newton methods are studied that terminate in a finite number of steps when applied to quadratic functions (quadratic finite termination). See [29, 33] and references therein. In this section, extending the analogous result obtained in [29] for $L$-BFGS, we will introduce conditions on $\widetilde{B}_{k}$ (see (58)) which endow the $\mathcal{S} B F G S$-type methods with the quadratic finite termination property.

Let us consider a pd matrix $A$ and the problem

$$
\min _{\mathbf{x} \in \mathbb{R}^{n}} f(\mathbf{x}) \text { where } f(\mathbf{x}):=\frac{1}{2} \mathbf{x}^{T} A \mathbf{x}-\mathbf{x}^{T} \mathbf{b} .
$$

In order to solve Problem (57) consider the following Algorithm 4 which is the $\mathcal{S}$ version of Algorithm 1 where we use the exact line search and where we set $H_{k}=B_{k}^{-1}, \widetilde{H}_{k}=\widetilde{B}_{k}^{-1}$ and $\phi=0$ (in Line 8 we have the Sherman-Morrison representation of $H_{k+1}=B_{k+1}^{-1}$ ).

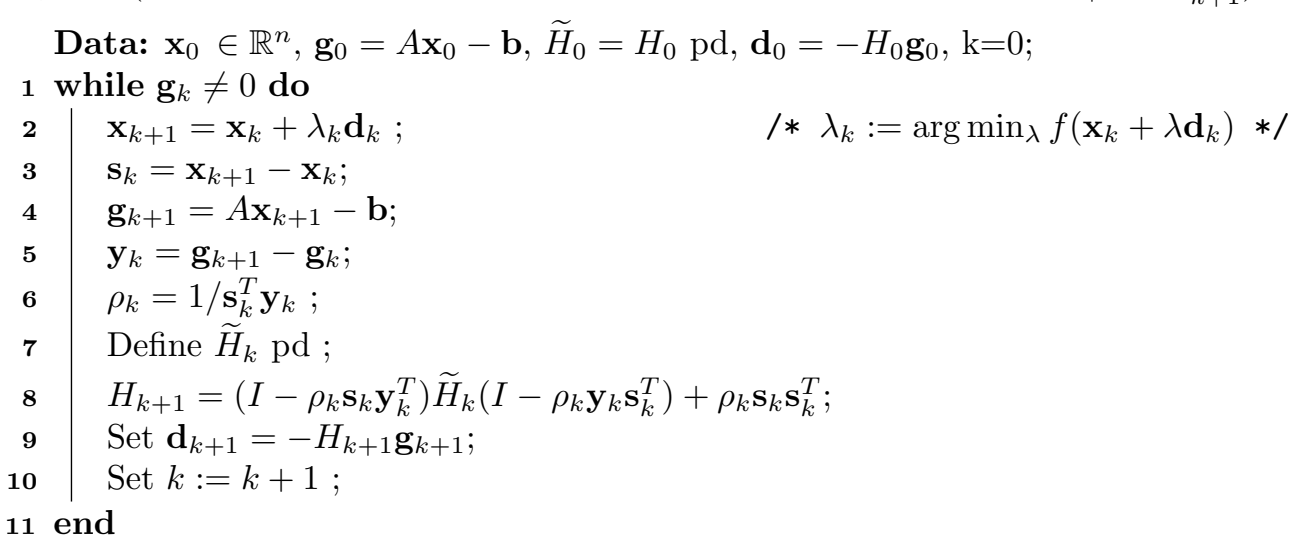

Algorithm 4: $B F G S$-type for quadratic problems

Theorem 4. Let us consider Algorithm 4. If

$$
\widetilde{H}_{k} \mathbf{g}_{k+1}=\beta_{k} H_{0} \mathbf{g}_{k+1} \text { for some } \beta_{k} \neq 0,
$$

then we have :

$$
\begin{gathered}
\mathbf{g}_{k+1}^{T} \mathbf{s}_{j}=0 \text { for all } j=0, \ldots, k ; \\
\mathbf{s}_{k+1}^{T} A \mathbf{s}_{j}=0 \text { for all } j=0, \ldots, k ; \\
\operatorname{Span}\left\{\mathbf{s}_{0}, \ldots, \mathbf{s}_{k+1}\right\}=\operatorname{Span}\left\{H_{0} \mathbf{g}_{0}, \ldots, H_{0} \mathbf{g}_{k+1}\right\} ;
\end{gathered}
$$

Proof. By induction. The case $k=0$ can be easily verified. Let us suppose the thesis true for $k=0, \ldots, \widehat{k}-1$ and prove it for $k=\widehat{k}$. Let us prove $(59): \mathbf{g}_{\widehat{k}+1}^{T} \mathbf{s}_{\widehat{k}}=0$ since we are using exact line search; if $j<\widehat{k}$ we have

$$
\mathbf{g}_{\widehat{k}+1}^{T} \mathbf{s}_{j}=\mathbf{g}_{\widehat{k}}^{T} \mathbf{s}_{j}+\mathbf{y}_{\widehat{k}}^{T} \mathbf{s}_{j}=\mathbf{g}_{\widehat{k}}^{T} \mathbf{s}_{j}+\mathbf{s}_{\widehat{k}}^{T} A \mathbf{s}_{j}=0
$$


by induction hypothesis. To prove (60) observe that for $j<\widehat{k}$

$$
\begin{aligned}
& \mathbf{s}_{\widehat{k}+1}^{T} A \mathbf{s}_{j}=-\lambda_{\widehat{k}+1} \mathbf{g}_{\widehat{k}+1}^{T} H_{\widehat{k}+1} \mathbf{y}_{j}= \\
& -\lambda_{\widehat{k}+1} \mathbf{g}_{\widehat{k}+1}^{T}\left(\left(I-\rho_{\widehat{k}} \mathbf{s}_{\widehat{k}} \mathbf{y}_{\widehat{k}}^{T}\right) \widetilde{H}_{\widehat{k}}\left(I-\rho_{\widehat{k}} \mathbf{y}_{\widehat{k}} \mathbf{s}_{\widehat{k}}^{T}\right)+\rho_{\widehat{k}} \mathbf{s}_{\widehat{k}} \mathbf{s}_{\widehat{k}}^{T}\right) \mathbf{y}_{j}= \\
& -\lambda_{\widehat{k}+1} \mathbf{g}_{\widehat{k}+1}^{T} \widetilde{H}_{\widehat{k}} \mathbf{y}_{j}=-\beta_{\widehat{k}} \lambda_{\widehat{k}+1} \mathbf{g}_{\widehat{k}+1}^{T} H_{0} \mathbf{y}_{j}=0
\end{aligned}
$$

where the third equality follows observing that $\mathbf{g}_{\widehat{k}+1}^{T} \mathbf{s}_{\widehat{k}}=0$ and that $\mathbf{s}_{\widehat{k}}^{T} \mathbf{y}_{j}=0$ for $j<\widehat{k}$ by induction hypothesis; the fourth equality follows by (58); the last equality follows observing that, since $\mathbf{g}_{\widehat{k}+1}^{T} \mathbf{s}_{\widehat{i}}=0$ for all $j=0, \ldots, \widehat{k}$ and $\operatorname{Span}\left\{\mathbf{s}_{0}, \ldots, \mathbf{s}_{\widehat{k}}\right\}=\operatorname{Span}\left\{H_{0} \mathbf{g}_{0}, \ldots, H_{0} \mathbf{g}_{\widehat{k}}\right\}$ by induction hypothesis, it holds that

$$
\mathbf{g}_{\widehat{k}+1}^{T} H_{0} \mathbf{g}_{j}=0 \text { for all } j=0, \ldots, \widehat{k} .
$$

Now let us consider the case $j=\widehat{k}$. Since $\mathbf{s}_{\widehat{k}+1}=-\lambda_{\widehat{k}+1} H_{\widehat{k}+1} \mathbf{g}_{\widehat{k}+1}$, by direct computation using the definition of $H_{\widehat{k}+1}$, it can be verified that $\mathbf{s}_{\widehat{k}+1}^{T} A \mathbf{s}_{\widehat{k}}=\mathbf{s}_{\widehat{k}+1}^{T} \mathbf{y}_{\widehat{k}}=0$. Let us prove now (61) : we have

$$
\begin{aligned}
& \mathbf{s}_{\widehat{k}+1}=-\lambda_{\widehat{k}+1} H_{\widehat{k}+1} \mathbf{g}_{\widehat{k}+1}=-\lambda_{\widehat{k}+1} \widetilde{H}_{\widehat{k}} \mathbf{g}_{\widehat{k}+1}+\lambda_{\widehat{k}+1} \rho_{\widehat{k}} \mathbf{y}_{\widehat{k}}^{T} \widetilde{H}_{\widehat{k}} \mathbf{g}_{\widehat{k}+1} \mathbf{s}_{\widehat{k}}= \\
& -\beta_{\widehat{k}} \lambda_{\widehat{k}+1} H_{0} \mathbf{g}_{\widehat{k}+1}+\lambda_{\widehat{k}+1} \rho_{\widehat{k}} \mathbf{y}_{\widehat{k}}^{T} \widetilde{H}_{\widehat{k}} \mathbf{g}_{\widehat{k}+1} \mathbf{s}_{\widehat{k}}
\end{aligned}
$$

and hence

$$
\operatorname{Span}\left\{H_{0} \mathbf{g}_{0}, \ldots, H_{0} \mathbf{g}_{\widehat{k}+1}\right\}=\operatorname{Span}\left\{\mathbf{s}_{0}, \ldots, \mathbf{s}_{\widehat{k}+1}\right\}
$$

since $\operatorname{Span}\left\{H_{0} \mathbf{g}_{0}, \ldots, H_{0} \mathbf{g}_{\widehat{k}}\right\}=\operatorname{Span}\left\{\mathbf{s}_{0}, \ldots, \mathbf{s}_{\widehat{k}}\right\}$ and $\left\{\mathbf{s}_{0}, \ldots, \mathbf{s}_{\widehat{k}+1}\right\}$ are linearly independent since they are $A$-conjugate.

Corollary 2. If the pd matrices $\widetilde{H}_{k}$ satisfy hypothesis of Theorem 4, then Algorithm 4 generates the same iterates as the Conjugate Gradient method preconditioned with $H_{0}$ and hence it converges in at most $n$ steps.

Proof. Analogous to the proof of Corollary 2.3 in [29], observing that under hypothesis of Theorem 4 conditions (59), (60) and (61) hold for Algorithm 4.

Interestingly enough, using the above corollary it can be shown that the iterates of $\mathrm{Al}$ gorithm 4 coincide with those from BFGS and $L$-BFGS since they all coincide with the Preconditioned Conjugate Gradient (see [33, 29]).

We can now prove that the convergence condition (39) and the quadratic termination condition (58) can be verified simultaneously if $\widetilde{B}_{k}^{-1}=\widetilde{H}_{k}=\mathcal{L}_{B_{k}}^{-1}$ provided that $H_{0}$ in (58) is a multiple of the identity.

Lemma 4. For any pair of vectors $\mathbf{s}_{k}, \mathbf{g}_{k+1}$ and pd matrix $B_{k}$ generated by Algorithm 4 with $H_{0}=I$, there exists a low complexity orthogonal matrix $L_{k}$ and hence a matrix algebra $\mathcal{L}^{(k)}=s d L_{k}$ such that

$$
\begin{aligned}
\mathcal{L}_{B_{k}}^{(k)} \mathbf{s}_{k} & =B_{k} \mathbf{s}_{k}, \\
\mathcal{L}_{B_{k}}^{(k)} \mathbf{g}_{k+1} & =\alpha_{k} \mathbf{g}_{k+1} \text { for some } \alpha_{k} \neq 0 .
\end{aligned}
$$

$L_{k}$ can be effectively constructed as the product of at most three Householder matrices.

Proof. For the sake of simplicity we use, in the following, the symbols $L$ and $\mathcal{L}$ in place of $L_{k}$ and $\mathcal{L}^{(k)}$.

1. Case $B_{k} \mathbf{s}_{k}=\gamma \mathbf{s}_{k}$.

From Theorem 4 we have $\mathbf{g}_{k+1}^{T} \mathbf{s}_{k}=0$. Any orthogonal matrix $L$ which has among its columns $\mathbf{s}_{k} /\left\|\mathbf{s}_{k}\right\|$ and $\mathbf{g}_{k+1} /\left\|\mathbf{g}_{k+1}\right\|$ is such that, defining $\mathcal{L}=\operatorname{sd} L, \mathcal{L}_{B_{k}}$ satisfies conditions in (66) (the columns of $L$ are eigenvectors of any matrix in $\mathcal{L}$ ). One of such orthogonal matrix $L$ can be constructed as the product of two orthogonal Householder matrices (see Lemma 5 in Appendix and see [13] for more details).

2. Case $B_{k} \mathbf{s}_{k} \neq \gamma \mathbf{s}_{k}$.

Any matrix $L$ in (52) with $m=2$ satisfies $\mathcal{L}_{B_{k}} \mathbf{s}_{k}=B_{k} \mathbf{s}_{k}$ if $\mathcal{L}=\operatorname{sd} L$; it is then enough to consider a matrix $L$ where $\mathbf{g}_{k+1} /\left\|\mathbf{g}_{k+1}\right\|$ is chosen to be one of the vectors $\mathbf{q}_{i}$; observe that this can be done since, from Theorem $4, \mathbf{g}_{k+1}^{T} \mathbf{s}_{k}=0=\mathbf{g}_{k+1}^{T} \mathbf{g}_{k}$ (see (64) with $\left.H_{0}=I\right)$ and since the first two columns of $L$ in (52), namely $V_{2} Q \mathbf{e}_{1}$ and $V_{2} Q \mathbf{e}_{2}$, are suitable linear combinations of $\mathbf{s}_{k}$ and $B_{k} \mathbf{s}_{k}=-\lambda_{k} \mathbf{g}_{k}$ (see the proof of Theorem 3 with $m=2$ and $\mathbf{s}_{k}, B_{k}$ in the roles of $\mathbf{s}$ and $A$ respectively). An orthogonal matrix $L$ with three columns fixed as $V_{2} Q \mathbf{e}_{1}, V_{2} Q \mathbf{e}_{2}$ and $\mathbf{g}_{k+1} /\left\|\mathbf{g}_{k+1}\right\|$, can be constructed as the product of three orthogonal Householder matrices (see Lemma 5 in Appendix and see [13] for more details). 


\section{A convergent $\mathcal{L}^{(k)} \mathrm{QN}$ method with quadratic termi- nation property}

The $\mathcal{L}^{(k)} Q N$ scheme that we consider in this section combines the results obtained in Section 3 for the $\mathcal{S}$ ecant scheme with $\phi=0$ and in Section 6 for quadratic termination, setting in both $\widetilde{B}_{k}=\mathcal{L}_{B_{k}}^{(k)}$. In particular it combines the convergence result stated in Theorem 1 for general non linear problems with the quadratic termination result obtained in Theorem 4. The main motivation for this choice can be traced to the key observation that in this way the resulting method coincides, as already pointed out in Section 6, with BFGS and $L-B F G S$ when applied on quadratic problems using exact line search.

\subsection{The proposed method}

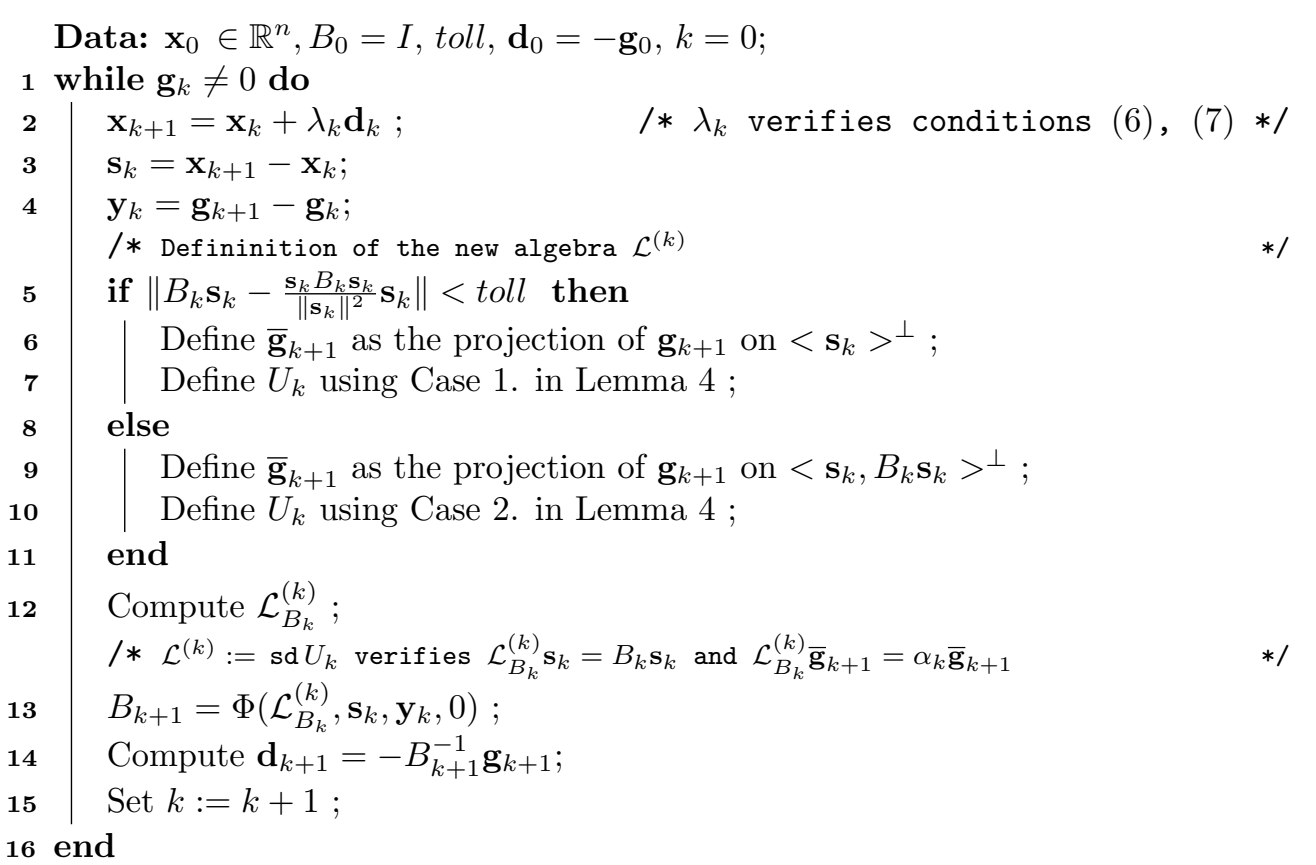

Algorithm 5: A convergent $\mathcal{L}^{(k)} \mathrm{QN}$ method with quadratic termination property verified if exact line search is used.

Observe that the applicability of Lemma 4, and hence the existence of the orthogonal matrices $U_{k}$ at lines 7 and 10 of Algorithm 5, are guaranteed by the definition of $\mathbf{g}_{k+1}$. Indeed, in Lemma 4 , where $f$ is quadratic, $\mathbf{g}_{k+1}$ is orthogonal to $\mathbf{s}_{k}$ and to $B_{k} \mathbf{s}_{k}$. When $f$ is not quadratic, $\mathbf{g}_{k+1}$ has to be replaced by the vector $\overline{\mathbf{g}}_{k+1}$ which is, by construction, orthogonal to both $\mathbf{s}_{k}$ and $B_{k} \mathbf{s}_{k}$. In particular, to perform Line 10 of Algorithm 5, one computes the projection of $\mathbf{g}_{k+1}$ on the space $<\mathbf{s}_{k}, B_{k} \mathbf{s}_{k}>^{\perp}$, that is, $\overline{\mathbf{g}}_{k+1}:=\left(I-V V^{T}\right) \mathbf{g}_{k+1}$ being $V:=\left[\mathbf{v}_{1} \mid \mathbf{v}_{2}\right]$ an orthonormal basis of $\left\langle\mathbf{s}_{k}, B_{k} \mathbf{s}_{k}>\right.$, and then apply Lemma 4 to $B_{k}=\Phi\left(\mathcal{L}_{B_{k-1}}^{(k-1)}, \mathbf{s}_{k-1}, \mathbf{y}_{k-1}, 0\right), \mathbf{s}_{k}$ and $\overline{\mathbf{g}}_{k+1}$, to obtain $U_{k}:=\mathcal{H}\left(\mathbf{h}_{3}^{(k)}\right) \mathcal{H}\left(\mathbf{h}_{2}^{(k)}\right) \mathcal{H}\left(\mathbf{h}_{1}^{(k)}\right)$ (see, moreover, Lemma 5 in the Appendix). For Line 7, proceed analogously; in this case $U_{k}:=\mathcal{H}\left(\mathbf{h}_{2}^{(k)}\right) \mathcal{H}\left(\mathbf{h}_{1}^{(k)}\right)$. Regarding Line 12 observe that, as in Algorithm 3, they consist in computing the eigenvalues of $\mathcal{L}_{B_{k}}^{(k)}$ by (56).

\subsection{Complexity}

An analogous analysis as in Section 5.2 permits to infer that the computational complexity of Algorithm 5 is $O(n)$ in space and time. Assuming that the matrices $U_{k}$ are always constructed according to Line 10 of Algorithm 5, a straightforward implementation of Algorithm 5 requires roughly $120 n$ multiplications and the storage of 17 vectors of length $n$.

\section{Numerical Results}

In our numerical experimentation we have used performance profiles (see [21]) in order to investigate and compare the numerical behavior of Algorithm 3 with $\phi=0$ (refinement of the method introduced in [12]), Algorithm 5, DQN [9], $\mathcal{H} Q N[4,16]$ and $L-B F G S$ with $M=5$ and $M=30$ [22]. The latter method, that has been implemented by the Poblano toolbox [23], has a computational cost of roughly $4 M n$ multiplications and requires the storage of $(4 M+2$ vectors to be implemented. We have tested the algorithms on a set of medium/large scale problems using the line-search routine provided in Poblano, i.e., the Moré-Thuente cubic interpolation line search (which implements the Strong-Wolfe conditions) enforcing 
the reproducibility of our results. In order to make a fair comparison we have used for all the algorithms the same stopping criteria as those from Poblano. The results have been obtained on a laptop running Linux with 16Gb memory and CPU Intel(R) Core(TM) i7-8th generation CPU with clock $2.00 \mathrm{GHz}$. The scalar code is written and executed in MATLAB R2018b. We have used the following parameters where the names of the variables are the same as those from Poblano (LineSearch_ftol $=\alpha$ in (6) and LineSearch_gtol $=\beta$ in (7)) :

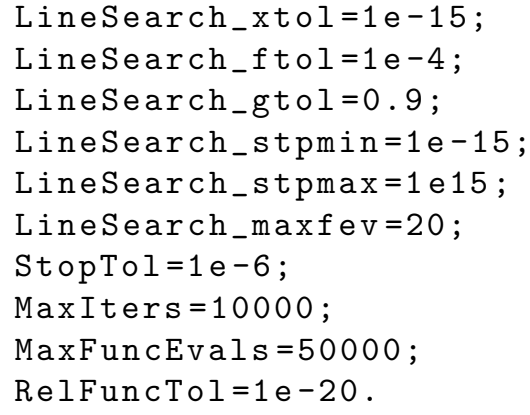

Finally, let us point out that, as in Poblano, the successful termination is achieved when $\left\|g_{k}\right\|_{2} / n \leq S t o p T o l$ being $n$ the dimension of the problem.

In all the following Figures " $\mathcal{L}^{(k)} \mathrm{QN}$ Sc" and " $\mathcal{L}^{(k)} \mathrm{QN}$ " indicate Algorithm 3 using, respectively, scaling as in Remark 7 or not. Analogously, " $\mathcal{L}^{(k)} \mathrm{QN}$ (q.t.) Sc" and " $\mathcal{L}^{(k)} \mathrm{QN}$ (q.t.) " indicate Algorithm 5 using, respectively, scaling as in Remark 7 or not.

\subsubsection{Experiment 1}

In this experiment we have chosen a problem set from CUTEst [26] where $L$-BFGS performs poorly. See Table 1 for the complete list of considered problems. In Figure 2 we show, using a logarithmic scale, the performance profiles of the selected solvers.
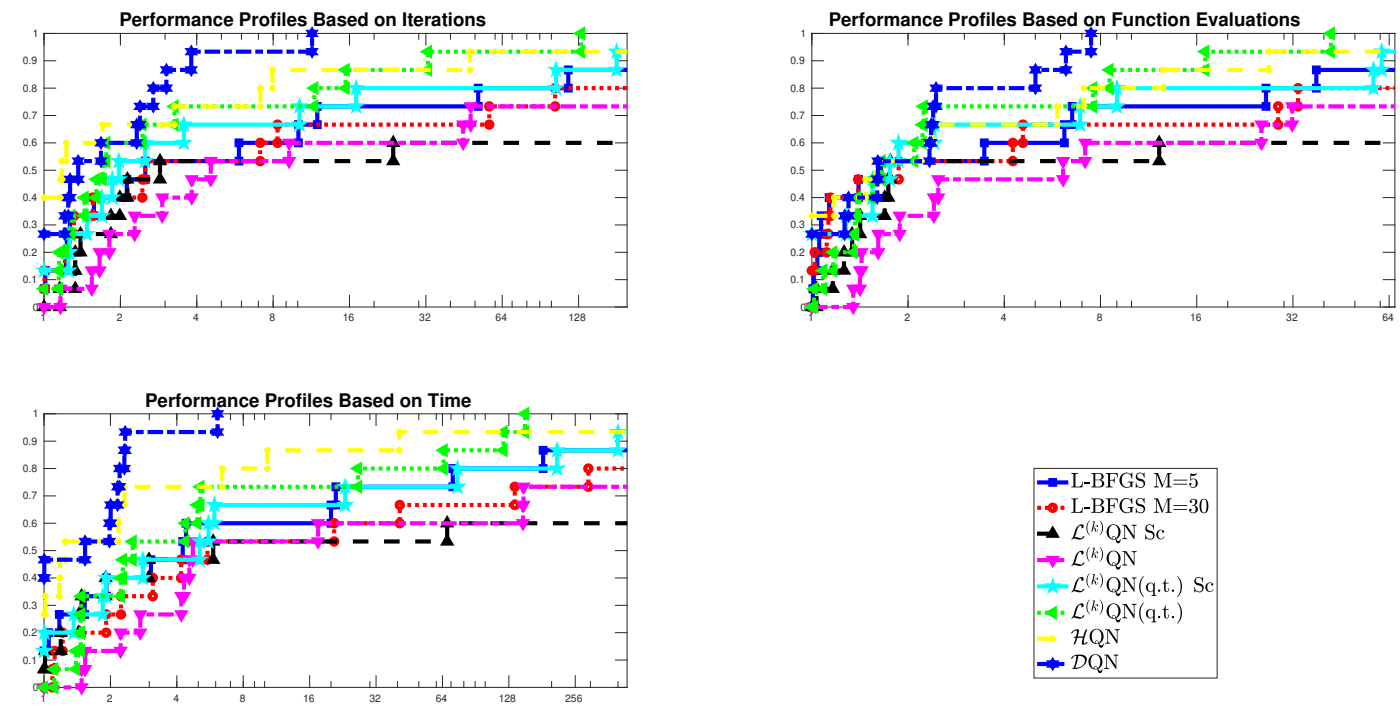

Figure 2: Performance profiles for Algorithm 3, Algorithm 5, DQN [9], $\mathcal{H} Q N[4,16]$ and $L-B F G S$ with $M=5$ and $M=30$ [22] on a set of 14 problems from CUTEst [26]. LineSearch_ftol $=1 \mathrm{e}-4$; LineSearch_gtol $=0.9$;

\begin{tabular}{|c|c|c|c|c|c|}
\hline Pro & Dim & $\begin{array}{l}\text { Table } 1 \\
\text { N.Z. }\end{array}$ & $\begin{array}{l}\text { roblem Set } \\
\text { Prob }\end{array}$ & D & N.Z. \\
\hline BROYDN7D & 5000 & 17497 & 8] MODBEALE & 20 & 99 \\
\hline CHAINWOO & 10000 & 19999 & 9] MSQRTALS & 4900 & 12007450 \\
\hline CURLY10 & 1000 & 10945 & 10] MSQRTBLS & 4900 & 12007450 \\
\hline EIGENBLS & 2550 & 3252525 & 11] NONCVXU2 & 10000 & 39987 \\
\hline EIGENCLS & 2652 & 3517878 & 12] SBRYND & 1000 & 6979 \\
\hline GENHUMPS & 5000 & 9999 & 13] TESTQUAD & 1000 & 1000 \\
\hline GENROSE & 500 & 999 & 14] TRIDIA & 5000 & 9999 \\
\hline
\end{tabular}




\subsubsection{Experiment 2}

In this experiment we have investigated the problem of approximating a given matrix $A \in$ $A^{m \times n}$ by a rank- $k$ approximation of the form $U V^{T}$, i.e., the function we wish to optimize is

$$
\min _{U \in \mathbb{R}^{m \times k}, V \in \mathbb{R}^{n \times k}}\left\|A-U V^{T}\right\|_{F}^{2} .
$$

Problem (67) arises in may applications (see for example [25] for applications connected with data mining). In particular, we focus on the dimensionality reduction problem $((m+n) k<<$ $m n)$ for MINST database [30]. The MINST test-set contains 10000 labeled handwritten digits from 0 to 9 stored as $28 \times 28$ matrices. For each class, we solve problem (67) where $A$ is a $m \times n=28^{2} \times$ class-size, being class-size the number of examples contained in the dataset for the considered digit. In Figures 3 and 4 we show, using a logarithmic scale, the performance profile of the selected solvers when $k=2^{6}$ and $k=2^{7}$. For details on the choice of the parameters see the preliminaries of this section; we use as $\mathbf{x}_{0}$ a random vector. In Table 2 we report the dimensions of the involved problems.
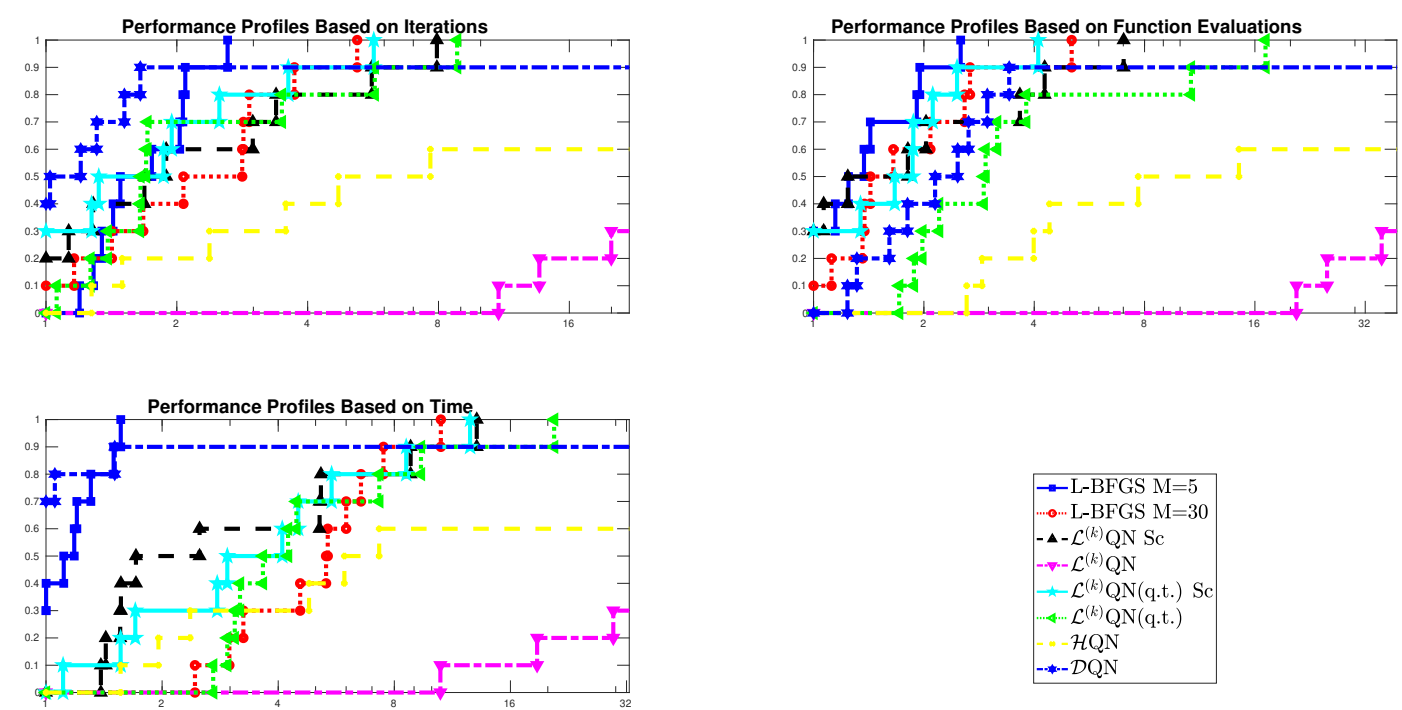

Figure 3: Performance profiles for Algorithm 3, Algorithm 5, DQN [9], $\mathcal{H} Q N[4,16]$ and $L-B F G S$ with $M=5$ and $M=30$ [22] when $k=2^{6}$. LineSearch_ftol=1e-4; LineSearch_gtol $=0.9$.
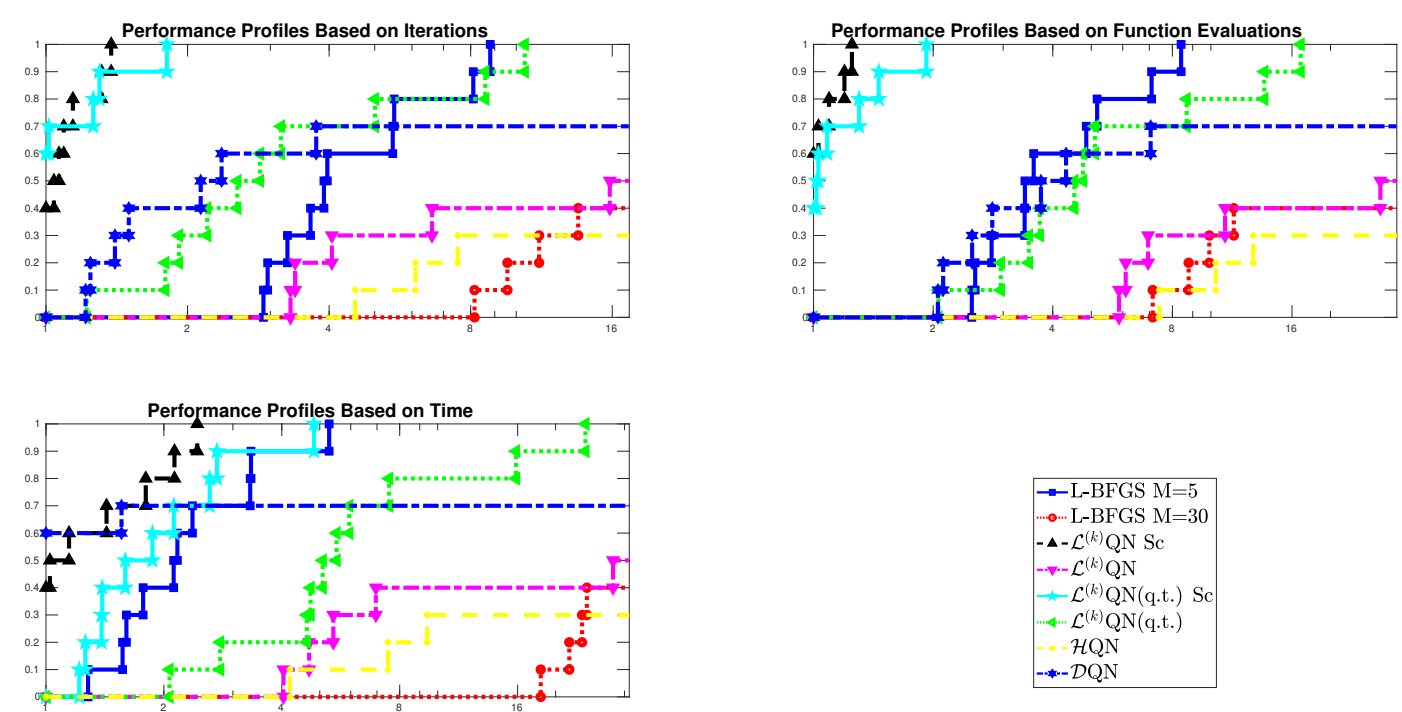

Figure 4: Performance profiles for Algorithm 3, Algorithm 5, DQN [9], $\mathcal{H} Q N[4,16]$ and $L-B F G S$ with $M=5$ and $M=30$ [22] when $k=2^{7}$. LineSearch_ftol=1e-4; LineSearch_gtol=0.9.

Table 2: MINST factorization: problems dimensions

\begin{tabular}{lllllllllll} 
Class/Rank & 0 & 1 & 2 & 3 & 4 & 5 & 6 & 7 & 8 & 9 \\
\hline$k=2^{6}$ & 112896 & 122816 & 116224 & 114816 & 113024 & 107264 & 111488 & 115968 & 112512 & 114752 \\
\hline$k=2^{7}$ & 225792 & 245632 & 232448 & 229632 & 226048 & 214528 & 222976 & 231936 & 225024 & 229504 \\
\hline
\end{tabular}




\subsection{Conclusions and future works}

In this work we have proposed and studied the convergence of novel optimization schemes $\mathcal{L}^{(k)} \mathrm{QN}$ obtained by generalizing the updates in the restricted Broyden class by means of projections of the Hessian approximations $B_{k}$ on adaptive low complexity matrix algebras $\mathcal{L}^{(k)}$, and in particular, we have studied in detail two new $B F G S$-type methods with theoretical guarantee of convergence.

The finite quadratic termination is not really relevant for general Quasi-Newton methods [29]. However, the numerical results presented in the previous subsections, see " $\mathcal{L}^{(k)} \mathrm{QN}$ " and " $\mathcal{L}^{(k)} \mathrm{QN}$ (q.t)" in Figures 2,3 and 4, confirm that if this property is added to $B F G S$ type algorithms, as in Algorithm 5, then we succeed in improving the performances of the basic $\mathcal{L}^{(k)} \mathrm{QN}$ scheme in Algorithm 3, which is a convergent refinement of the methods considered in $[12]$.

Moreover the numerical results show that, by an adaptive choice of the matrix algebras $\mathcal{L}^{(k)}$, the robustness of the existing fixed algebras $\mathcal{L} Q N$ methods, $\mathcal{D} Q N$ and $\mathcal{H} Q N$, can be overcome (see Figures 3 and 4), even though this does not guarantee the best performance in terms of Iteration, Function Evaluations or Execution Time (see Figure 2). Notice, moreover, that the methods $\mathcal{D Q N}$ and $\mathcal{H Q N}[4,9,24]$ are competitive for other classes of problems.

Now, in Experiment 1, the comparison of the proposed $\mathcal{L}^{(k)} \mathrm{QN}$ methods is not totally favorable. In fact, Figure 2 shows that the best performers are $\mathcal{D} Q N$ and $\mathcal{H} Q N$. However, the improved robustness of our proposal, already traceable in Experiment 1, is further underpinned by Experiment 2, where Algorithm 5 always reaches the required level of accuracy within the maximum number of allowed iterations, whereas $L-B F G S$ with $M=5$ and $M=30$ drastically changes its behavior when switching from rank $2^{6}$ to rank $2^{7}$. In this experiment, a straightforward implementation of our proposals does not guarantee to outperform $L-B F G S$ with $M=5$.

However, on this set of problems, the efficiency of our proposals is dramatically improved by introducing a self-scaling factor as outlined in Remark 7. In this case, see " $\mathcal{L}(k) \mathrm{QN}$ Sc" and " $\mathcal{L}^{(k)} \mathrm{QN}$ (q.t) Sc" in Figure 4, our proposals clearly outperform $L-B F G S$ with $M=5$ and $M=30$.

It is important to note that our proposals dot not require the choice of a problem dependent parameter as $M$ in $L-B F G S$ and that, in general, if $M$ is big, require less memory to be implemented.

By the above reasons, further investigation urges in order to understand if the new method could be a valid competitor of $L-B F G S$, in particular for those problems where large values of the parameter $M$ must be chosen in order to guarantee satisfactory performances (see also [28]) or for those problems where the computation of the gradient is expensive, as those coming from data science or optimal control (see, for example, $[5,14]$ ).

It is clear that $\mathcal{L}^{(k)} Q N$ methods should be also compared with the class of nonlinear conjugate gradient methods. Moreover, it would be important to understand if the matrices generated by means of our Quasi Newton-type updates could be useful as preconditioners for nonlinear conjugate gradient methods as in [10]. Of course, further investigation should be devoted, in future, in order to understand if the Broyden Class-version of Algorithm 3 or Algorithm 5 can produce better performances for $\phi \in(0,1)$. Last but not least, it could be interesting to understand if the results presented in this paper can be extended to the modified BFGS method for non-convex functions as in [31]. Finally the connections with Quasi-Newton Self-Scaling methods [35, 2] should be further explored.

\section{Acknowledgments}

We would like to thank the referees for their thorough reading of the manuscript, valuable suggestions and for pointing to relevant typos.

\section{Appendix 1: Householder Matrices}

The results contained in this section are borrowed from [13] and we refer the interested reader there for more details.

Definition 1 (Householder Orthogonal Matrix). Given a vector $\mathbf{p} \in \mathbb{R}^{n}$ define

$$
\mathcal{H}(\mathbf{p}):=I_{n}-\frac{2}{\|\mathbf{p}\|^{2}} \mathbf{p p}^{T} .
$$

Consider two vectors $\mathbf{v}, \mathbf{z} \in \mathbb{R}^{n}$. From direct computation one can check that defining 
$\mathbf{p}=\mathbf{v}-\frac{\|\mathbf{v}\|}{\|\mathbf{z}\|} \mathbf{z}$ with $\mathbf{z} \neq 0$, we have

$$
\mathcal{H}(\mathbf{p}) \mathbf{v}=\frac{\|\mathbf{v}\|}{\|\mathbf{z}\|} \mathbf{z}
$$

Lemma 5 ([13]). Consider $W=\left[\mathbf{w}_{1}|\ldots| \mathbf{w}_{s}\right] \in \mathbb{R}^{n \times s}, V=\left[\mathbf{v}_{1}|\ldots| \mathbf{v}_{s}\right] \in \mathbb{R}^{n \times s}$ of full rank and such that $s \leq n, W^{T} W=V^{T} V$. Then there exist $\mathbf{h}_{1}, \ldots, \mathbf{h}_{s} \in \mathbb{R}^{n},\left\|\mathbf{h}_{i}\right\|=\sqrt{2}$, such that the orthogonal matrix $U=\mathcal{H}\left(\mathbf{h}_{s}\right) \cdots \mathcal{H}\left(\mathbf{h}_{1}\right)$, product of $s$ Householder matrices, satisfies the following identities

$$
U \mathbf{w}_{i}=\mathbf{v}_{i} \text { for all } i \in\{1, \ldots, s\} .
$$

The vectors $\mathbf{h}_{i}$ for $i \in\{1, \ldots, s\}$ can be obtained by setting:

$$
\begin{aligned}
\widetilde{\mathbf{h}}_{i} & :=\mathcal{H}\left(\mathbf{h}_{i-1}\right) \cdots \mathcal{H}\left(\mathbf{h}_{1}\right)\left(\mathbf{w}_{i}-\mathbf{w}_{i-1}\right)-\left(\mathbf{v}_{i}-\mathbf{v}_{i-1}\right), \\
\mathbf{h}_{i} & :=\left(\sqrt{2} /\left\|\widetilde{\mathbf{h}}_{i}\right\|\right) \widetilde{\mathbf{h}}_{i}
\end{aligned}
$$

(where we set $\mathbf{h}_{0}=\mathbf{w}_{0}=\mathbf{v}_{0}=\mathbf{0}$ ). If $s=n$ we have $\mathbf{h}_{n}=\mathbf{0}$ or $\mathbf{h}_{n}=\frac{\sqrt{2}}{\left\|\mathbf{v}_{n}\right\|} \mathbf{v}_{n}$. The cost of the computation of the $\mathbf{h}_{i}$ for $i=1, \ldots, s$ is:

$$
[s(s-1) n+s(2 n+1)] \text { mult. }+[(s(s+2)-2) n+s(n-1)] \text { add. }+s \text { sq. roots. }
$$

Observe that when $\mathbf{w}_{i}=\mathbf{e}_{k_{i}}$ for $i=1, \ldots, s$, that is when $\mathbf{v}_{1}, \ldots, \mathbf{v}_{s}$ are orthonormal and we are interested to construct an orthogonal $U$ with $s$ columns fixed as $\mathbf{v}_{1}, \ldots, \mathbf{v}_{s}$, it is possible to save $(s-1) n$ mult. and $(3 s-2) n$ add..

Proof. The explicit expression of the $\mathbf{h}_{i}$ in (68) is obtained by applying the techniques for their construction introduced in [13].

\section{Appendix 2: details on Theorem 1}

In order to prove inequality (38) it is enough to prove that:

Lemma 6. There exists $c_{3}$ constant with respect to $j$ and depending only on $s$ and $M$ such that

$$
\gamma((j+1-s)+1)^{n} \leq c_{3}^{j+1-s} \text { for all } j \geq s, \text { where } \gamma:=\left(\frac{c_{1}}{n}\right)^{n} \frac{1}{\operatorname{det} B_{s}}
$$

(of course, such $c_{3}$ turns out to be greater than 1 ).

In fact, once Lemma 6 is proved, the constant $c_{2}$ (constant with respect to $j$ ) for which (38) is verified, will be $c_{2}=2 c_{1} c_{3} /(1-\beta)$ (note that $c_{2}$ depends only $s, M, \beta$ but not on $j$ ).

Proof. Fix $\widetilde{c}_{3}>1$. Note that the sequence of positive numbers

$$
\frac{\gamma((j+1-s)+1)^{n}}{\widetilde{c}_{3}^{j+1-s}} \text { for } j=s, s+1, \ldots
$$

converges to zero as $j \rightarrow+\infty$; thus there exists $j^{*} \geq s$ (depending on $s, M$ and $\widetilde{c}_{3}$ ) s.t.

$$
\gamma((j+1-s)+1)^{n} \leq \widetilde{c}_{3}^{j+1-s} \text { for all } j \geq j^{*} .
$$

Note also that for all $j \in\left\{s+1, \ldots, j^{*}-1\right\}$ we have

$$
\gamma((j+1-s)+1)^{n} \leq \gamma\left(j^{*}-s+1\right)^{n}
$$

and consider $\widehat{j} \geq j^{*}$ s.t. $\gamma(\widehat{j}-s+1)^{n}>1\left(\widehat{j}\right.$ depends on $s, M, \gamma$ and $\left.\widetilde{c}_{3}\right)$. From (69) we have

$$
\gamma((j+1-s)+1)^{n} \leq \gamma(\widehat{j}-s+1)^{n} \leq\left(\gamma(\widehat{j}-s+1)^{n}\right)^{j+1-s}
$$

for all $j \in\left\{s, s+1, \ldots j^{*}-1\right\}$.

Collecting the above results, we can conclude that

$$
\gamma((j+1-s)+1)^{n} \leq c_{3}^{j+1-s} \text { for all } j \geq s
$$

where $c_{3}:=\max \left\{\widetilde{c}_{3}, \gamma(\hat{j}-s+1)^{n}\right\}\left(c_{3}>1\right.$ and depends on $s, M$ and $\left.\widetilde{c}_{3}\right)$.

Finally note that, once $\widetilde{c}_{3}$ is fixed, it is clear that $c_{3}$ depends only on $s, M$. 
In order to prove inequality $\left(38_{1}\right)$, define $a_{k}:=\left(1-\phi-\psi_{k} \phi\right)\left\|\mathbf{g}_{k}\right\|^{2} / \mathbf{s}_{k}^{T}\left(-\mathbf{g}_{k}\right)>0$. We know that $\lim _{k \rightarrow+\infty} a_{k}=+\infty$ and we have to show that there exists $j^{*} \geq s$ such that

$$
\prod_{k=s}^{j} a_{k}>c_{2}^{j+1-s} \text { for all } j \geq j^{*} .
$$

If $a_{k} \geq c_{2}$ for all $k \geq s$, since it must be $a_{k}>c_{2}$ for infinite indexes $k$, then the thesis is obvious. So assume that there exists some index $k$ such that $a_{k}<c_{2}$. Let $r \geq s$ be such that $a_{k}>c_{2}$ for all $k>r$. Note that $c_{2}>\min _{k=s, \ldots, r} a_{k}$. Set

$$
t:=\left(\frac{c_{2}}{\min _{k=s, \ldots, r} a_{k}}\right)^{r+1-s}>1 .
$$

Let $j^{*}>r+1$ be such that $a_{k} \geq t c_{2}$ for all $k \geq j^{*}$. Then we have

$$
\begin{aligned}
& \prod_{k=s}^{j^{*}} a_{k}=\left(\prod_{k=s}^{r} a_{k}\right)\left(\prod_{k=r+1}^{j^{*}-1} a_{k}\right) a_{j^{*}}> \\
& \left(\min _{k=s, \ldots, r} a_{k}\right)^{r-s+1} c_{2}^{j^{*}-r-1} t c_{2}= \\
& \left(\min _{k=s, \ldots, r} a_{k}\right)^{r-s+1}\left(\frac{c_{2}}{\min _{k=s, \ldots, r} a_{k}}\right)^{r-s+1} c_{2}^{j^{*}-r}=c_{2}^{j^{*}-s+1},
\end{aligned}
$$

i.e., $\prod_{k=s}^{j^{*}} a_{k}>c_{2}^{j^{*}-s+1}$. Thus we obtain (71) since $a_{k} \geq t c_{2}>c_{2}$ for $k>j^{*}$.

\section{References}

[1] M. Al-Baali. Analysis of a family of self-scaling quasi-newton methods. Dept. of Mathematics and Computer Science, United Arab Emirates University, Tech. Report, 1993.

[2] M. Al-Baali. Global and superlinear convergence of a restricted class of self-scaling methods with inexact line searches, for convex functions. Comput. Optim. Appl., 9(2):191-203, 1998.

[3] N. Andrei. A double-parameter scaling broyden-fletcher-goldfarb-shanno method based on minimizing the measure function of byrd and nocedal for unconstrained optimization. J. Optim. Theory Appl., 178(1):191-218, 2018.

[4] A. Bortoletti, C. Di Fiore, S. Fanelli, and P. Zellini. A new class of quasi-newtonian methods for optimal learning in MLP-networks. IEEE Trans. Neural Netw., 14(2):263273, Mar 2003.

[5] L. Bottou, F. E. Curtis, and J. Nocedal. Optimization methods for large-scale machine learning. Siam Rev., 60(2):223-311, 2018.

[6] R. H. Byrd, S. L. Hansen, J. Nocedal, and Y. Singer. A stochastic quasi-newton method for large-scale optimization. SIAM J. Optim., 26(2):1008-1031, 2016.

[7] R. H. Byrd and J. Nocedal. A tool for the analysis of quasi-newton methods with application to unconstrained minimization. SIAM J. Numer. Anal., 26(3):727-739, 1989.

[8] R. H. Byrd, J. Nocedal, and Y.-X. Yuan. Global convergence of a class of Quasi-Newton methods on convex problems. SIAM J. Numer. Anal., 24(5):1171-1190, 1987.

[9] J. F. Cai, R. H. Chan, and C. Di Fiore. Minimization of a detail-preserving regularization functional for impulse noise removal. J. Math. Imaging Vision, 29(1):79-91, 09 2007.

[10] A. Caliciotti, G. Fasano, and M. Roma. Novel preconditioners based on quasi-newton updates for nonlinear conjugate gradient methods. Optim. Lett., 11(4):835-853, 2017.

[11] S. Cipolla, C. Di Fiore, and F. Tudisco. Euler-Richardson method preconditioned by weakly stochastic matrix algebras: a potential contribution to Pagerank computation. Electron. J. Linear Algebra, 32:254-272, 2017.

[12] S. Cipolla, C. Di Fiore, F. Tudisco, and P. Zellini. Adaptive matrix algebras in unconstrained minimization. Linear Algebra Appl., 471(0):544 - 568, 2015.

[13] S. Cipolla, C. Di Fiore, and P. Zellini. Low complexity matrix projections preserving actions on vectors. Calcolo, 56(2):8, 2019. 
[14] S. Cipolla and F. Durastante. Fractional PDE constrained optimization: An optimizethen-discretize approach with L-BFGS and approximate inverse preconditioning. Appl. Numer. Math., 123:43-57, 2018.

[15] C. Di Fiore. Structured matrices in unconstrained minimization methods. Chap. in Contemporary Mathematics, pages 205-219, 2003.

[16] C. Di Fiore, S. Fanelli, F. Lepore, and P. Zellini. Matrix algebras in Quasi-Newton methods for unconstrained minimization. Numer. Math., 94(3):479-500, 2003.

[17] C. Di Fiore, S. Fanelli, and P. Zellini. Low-complexity minimization algorithms. Numer. Linear Algebra Appl., 12(8):755-768, 2005.

[18] C. Di Fiore, S. Fanelli, and P. Zellini. Low complexity secant quasi-newton minimization algorithms for nonconvex functions. J. Comput. Appl. Math., 210(1-2):167-174, 2007.

[19] C. Di Fiore, F. Lepore, and P. Zellini. Hartley-type algebras in displacement and optimization strategies. Linear Algebra Appl., 366:215-232, 2003.

[20] C. Di Fiore and P. Zellini. Matrix algebras in optimal preconditioning. Linear Algebra Appl., 335(1-3):1 - 54, 2001.

[21] E. D. Dolan and J. J. Moré. Benchmarking optimization software with performance profiles. Math. Program., 91(2):201-213, 2002.

[22] C. L. Dong and J. Nocedal. On the limited memory BFGS method for large scale optimization. Math. Program., 45(1-3):503-528, 081989.

[23] D. M. Dunlavy, T. G. Kolda, and E. Acar. Poblano v1. 0: A matlab toolbox for gradientbased optimization. Sandia National Laboratories, Albuquerque, NM and Livermore, CA, Tech. Rep. SAND2010-1422, 2010.

[24] A. Ebrahimi and G. Loghmani. B-spline curve fitting by diagonal approximation BFGS methods. Iran. J. Sci. Technol. Trans. A Sci., pages 1-12.

[25] L. Eldén. Numerical linear algebra in data mining. Acta Numer., 15:327-384, 2006.

[26] N. I. Gould, D. Orban, and P. L. Toint. Cutest: a constrained and unconstrained testing environment with safe threads for mathematical optimization. Comput. Optim. Appl., 60(3):545-557, 2015.

[27] R. A. Horn and C. R. Johnson. Matrix analysis, 2nd. Cambridge University press, 2013.

[28] L. Jiang, R. H. Byrd, E. Eskow, and R. B. Schnabel. A preconditioned L-BFGS algorithm with application to molecular energy minimization. Technical report, Colorado University at Boulder Dept. of Computer Science, 2004.

[29] T. G. Kolda, D. P. O'leary, and L. Nazareth. BFGS with update skipping and varying memory. SIAM J. Optim., 8(4):1060-1083, 1998

[30] Y. Lecun, L. Bottou, Y. Bengio, and P. Haffner. Gradient-based learning applied to document recognition. Proceedings of the IEEE, 86(11):2278-2324, 1998.

[31] D.-H. Li and M. Fukushima. A modified BFGS method and its global convergence in nonconvex minimization. J. Comput. Appl. Math., 129(1):15-35, 2001.

[32] C. Liu and S. A. Vander Wiel. Statistical Quasi-Newton: A new look at least change. SIAM J. Optim., 18(4):1266-1285, 2007.

[33] L. Nazareth. A relationship between the BFGS and conjugate gradient algorithms and its implications for new algorithms. SIAM J. Numer. Anal., 16(5):794-800, 1979.

[34] J. Nocedal and S. J. Wright. Numerical Optimization. Springer, 2nd edition, 2006

[35] J. Nocedal and Y.-x. Yuan. Analysis of a self-scaling Quasi-Newton method. Math. Program., 61(1-3):19-37, 1993.

[36] S. S. Oren and D. G. Luenberger. Self-scaling variable metric (SSVM) algorithms: Part i: Criteria and sufficient conditions for scaling a class of algorithms. Management Science, 20(5):845-862, 1974.

[37] M. J. D. Powell. Some global convergence properties of a variable metric algorithm for minimization without exact line searches. Nonlinear Program., SIAM-AMS Proc., 9:53-72, 1976 . 
[38] Y. Saad. Analysis of some Krylov subspace approximations to the matrix exponential operator. SIAM J. Numer. Anal., 29(1):209-228, Feb. 1992.

[39] Y. Saad. Numerical methods for large eigenvalue problems. SIAM, 2011. 